\title{
Transneptunian objects and Centaurs from light curves ${ }^{\star}$
}

\author{
R. Duffard, J. L. Ortiz, A. Thirouin, P. Santos-Sanz, and N. Morales
}

\author{
Instituto de Astrofísica de Andalucía - CSIC, Apt 3004, 18080 Granada, Spain \\ e-mail: duffard@iaa.es
}

Received 29 May 2009 / Accepted 6 July 2009

\begin{abstract}
Aims. We compile and analyze an extended database of light curve parameters scattered in the literature to search for correlations and study physical properties, including internal structure constraints.

Methods. We analyze a vast light curve database by obtaining mean rotational properties of the entire sample, determining the spin frequency distribution and comparing those data with a simple model based on hydrostatic equilibrium.

Results. For the rotation periods, the mean value obtained is $6.95 \mathrm{~h}$ for the whole sample, $6.88 \mathrm{~h}$ for the Trans-neptunian objects (TNOs) alone and $6.75 \mathrm{~h}$ for the Centaurs. From Maxwellian fits to the rotational frequencies distribution the mean rotation rates are $7.35 \mathrm{~h}$ for the entire sample, $7.71 \mathrm{~h}$ for the TNOs alone and $8.95 \mathrm{~h}$ for the Centaurs. These results are obtained by taking into account the criteria of considering a single-peak light curve for objects with amplitudes lower than 0.15 mag and a double-peak light curve for objects with variability $>0.15 \mathrm{mag}$. We investigate the effect of using different values other than $0.15 \mathrm{mag}$ for the transition threshold from albedo-caused light curves to shape-caused light curves. The best Maxwellian fits were obtained with the threshold between 0.10 and $0.15 \mathrm{mag}$. The mean light-curve amplitude for the entire sample is $0.26 \mathrm{mag}, 0.25 \mathrm{mag}$ for TNOs only, and 0.26 mag for the Centaurs. The Period versus $B-V$ color shows a correlation that suggests that objects with shorter rotation periods may have suffered more collisions than objects with larger ones. The amplitude versus $H_{v}$ correlation clearly indicates that the smaller (and collisionally evolved) objects are more elongated than the bigger ones.

Conclusions. From the model results, it appears that hydrostatic equilibrium can explain the statistical results of almost the entire sample, which means hydrostatic equilibrium is probably reached by almost all TNOs in the $H$ range $[-1,7]$. This implies that for plausible albedos of 0.04 to 0.20 , objects with diameters from $300 \mathrm{~km}$ to even $100 \mathrm{~km}$ would likely be in equilibrium. Thus, the great majority of objects would qualify as being dwarf planets because they would meet the hydrostatic equilibrium condition. The best model density corresponds to $1100 \mathrm{~kg} / \mathrm{m}^{3}$.
\end{abstract}

Key words. Kuiper belt - solar system: formation - techniques: photometric

\section{Introduction}

The belt of remnant planetesimals in the so-called Kuiper belt, whose existence was confirmed observationally in 1992 (Jewitt \& Luu 1993), is an important source of knowledge about the formation and early evolution of the Solar System. Features in the orbital distribution of the belt can provide important information about the early dynamical processes such as planetary migration, which was merely untested theories around 25 years ago (Fernandez \& Ip 1984) that now can be regarded as being well proven. The detailed architecture of the Kuiper belt might also be compatible with a very unstable phase when Jupiter and Saturn entered into resonance (Tsiganis et al. 2005; Morbidelli et al. 2005; Gomes et al. 2005) around 800 Myr after the Solar System formation. Although this particular dynamical scenario is still disputed, there is no question that the architecture of the transneptunian belt can offer invaluable clues about the dynamical mechanisms acting in the early Solar System. In addition to dynamical inferences from the orbital characteristics of the TNOs, much can be learnt about the physical processes that took place in the solar nebula by means of the study of the physical properties of TNOs and related groups of objects. Since the TNOs are understood to be mixtures of ices and rocks from

\footnotetext{
* Lightcurves are available in electronic form at the CDS via anonymous ftp to cdsarc.u-strasbg.fr (130.79.128.5) or via http://cdsweb.u-strasbg.fr/cgi-bin/qcat?]/A+A/505/1283
}

which the Centaurs and ultimately the Jupiter family comets (JFCs) originate (Jewitt 2004), ground and space-based observations of all these populations can give us clues about the physics of the outer Solar System. From the "Nice" dynamical model (Tsiganis et al. 2005; Morbidelli et al. 2005; Gomes et al. 2005), it is also likely that the Jupiter trojans are closely related to the TNOs (Morbidelli et al. 2005), but this is not yet well established.

These objects are regarded as the least evolved objects in the Solar System, but they have experienced many different types of physical processes that have altered them considerably. These processes range from collisions, to space weathering. The collisional processes have probably left clear imprints in both the spin distribution and in the light-curve amplitude distribution of TNOs and Centaurs. The currently accepted theory based on the study of Davis \& Farinella (1997) on collisional evolution of the TNOs is that objects of sizes larger than $100 \mathrm{~km}$ are very slightly collisionally evolved, whereas the smaller fragments have experienced more collisional events and hence should have their rotations and shapes more significantly altered. This picture is also consistent with newer collisional evolution models (Benavidez \& Campo Bagatin 2009). However, detailed modelling of the evolution of the spin rates related to collisional models has not yet been carried out for the transneptunian belt.

Due to the dedicated efforts of two main groups studying short-term variability (Gutierrez et al. 2001; Ortiz et al. 2003b,a, 
2004, 2006, 2007; Moullet et al. 2008; Duffard et al. 2008; Sheppard 2007; Sheppard \& Jewitt 2002; Sheppard et al. 2000) and occasional contributions by other authors, a database of about 40 rotation periods and 80 light-curve amplitudes could be extracted from the literature in 2008. Thus, we now have sufficient numbers of TNOs light curves to start analyzing them from a statistical point of view. In a review by Sheppard et al. (2008), those data were compiled and some conclusions were outlined. After that compilation, a new set of light curves from 29 TNOs and Centaurs became available (Thirouin et al. 2009) and those authors also presented evidence of possible biases that can be present in the light curve database gathered from the literature.

We also developed a simple model to interpret rotation rates and light-curve amplitudes together, to obtain information about densities and tensile strengths. Instead of analyzing each body separately and trying to retrieve information about its density and cohesion by using its spin rate and light-curve amplitude (and an assumed viewing angle), we address the mean internal properties by analyzing the population as a whole. In other words, we developed a model that can simultaneously interpret the distribution of spin rates and the distribution of light-curve amplitudes.

In this paper, light curve parameters of a vast sample are compiled and analyzed. We focus on the analysis of the period and shape distribution presented in Sect. 2. Then, with the current data, we complete a correlation analysis with several orbital parameters in Sect. 3. Internal properties of the TNOs are derived by applying a simple model in Sect. 4, a discussion of the results is presented in Sect. 5. Finally, the conclusions are presented in Sect. 6.

\section{Period and light-curve amplitude distributions}

One important current limitation is that the light curves of this kind of objects are accessible only with medium-size $(2 \mathrm{~m})$ or larger telescopes. The telescope time required for this type of program is difficult to obtain, and the observed objects are the brightest and largest ones. Only several light curves were observed with the Hubble Space Telescope (HST) reaching a lower limit of 20-100 km in diameter (Trilling \& Bernstein 2006). The current Kuiper belt object (KBO) absolute magnitude for which a good quality light curve can be derived (with a $2 \mathrm{~m}$ telescope), is $H<9$ for TNOs and $H<11$ for Centaurs. Smaller KBOs can only be observed with space telescopes.

Using the literature and our recent results (Thirouin et al. 2009), it is possible to create a database of light curves with rotational periods and peak-to-peak amplitudes. The special case of Pluto, whose spin rate has been altered by tidal dissipation caused by Charon, has been removed from the sample. A compilation of 102 objects, updated on March 2009, is presented in Table 1. From that list, only 72 objects have good enough period and light-curve amplitude estimates.

When looking and analyzing the current TNO and Centaur light curves, the first noticeable characteristic in most of the derived light curves is that the amplitudes are very small $(<0.15 \mathrm{mag})$. The criterium of placing a limit of $0.15 \mathrm{mag}$ was introduced by Sheppard \& Jewitt (2002) and used by other authors (Lacerda \& Luu 2006; Ortiz et al. 2003b,a) to distinguish the "photometrically flat" curves. The brightness changes are probably caused by the combined effect of rotation, irregular shape and/or albedo markings on the surface. Thus, the abundance of low amplitude light curves is indicative of a large abundance of quasi-spherical bodies with homogeneous surfaces. However, this is to some degree a selection effect because, as mentioned above, the true sample is dominated by "bright" objects, which are presumably the largest ones and nearly spherical.

An example of the kind of light curve studied here is presented in Fig. 1. In this example, data of the object $2003 \mathrm{VS}_{2}$ over several nights was observed and the composite light curve was compiled to determine the rotational period and amplitude. Unfortunately, it is not always possible to obtain a well determined period and/or amplitude. Authors sometimes place a constraint on the values of periods and/or amplitudes, and we note that the literature is populated by these kinds of results. In Table 1, we list all objects with published rotational period and/or light curve amplitude or constraints in one of these values. In any case, for all the considerations in the paper only the objects with a well determined period/amplitude pair are taken into account. That is the case for 72 objects. In case of multiple determinations of the period and/or amplitude, the criteria selected the preferred value by the author who published that value. If no preferred value is mentioned, the mean of the listed values was used.

\subsection{Amplitude distribution}

The KBOs albedo may not be uniform across their surfaces, causing the apparent magnitude to vary as the different albedo markings on the KBOs surface rotate in and out of our line of sight. Albedo or surface variations of the object are usually responsible for less than a $20 \%$ difference from maximum to minimum brightness of an object (Degewij et al. 1979; Magnusson 1991) in the asteroid case. In Thirouin et al. (2009), it is estimated that the typical TNO variability caused by albedo markings is around $0.1 \mathrm{mag}$. The reasons for the apparently small amount of variability due to albedo markings on the TNO surfaces compared to that of the asteroids may be related to the icier surfaces of the TNOs than those of the asteroids. However, also the processing of the surfaces is different, because different mechanisms can take place. Thus, it is unsurprising that the surface reflectance variability may be different for TNOs than for asteroids. The majority of low-amplitude light curves of large objects are believed to be caused by this kind of surface variations (Sheppard et al. 2008).

On the other hand, shape variations or elongation of an object will cause the effective radius of an object to our line of sight to change as the KBO rotates. Smaller KBOs are expected to be structurally elongated. To date, few small $(20-100 \mathrm{~km}) \mathrm{KBOs}$ have been observed with rotational variations, and they appear to have larger light curve amplitudes (Trilling \& Bernstein 2006).

In Fig. 2, we show the percentage of objects that have an amplitude value within a specific peak-to-peak amplitude (in $0.1 \mathrm{mag}$ bin) of the well determined 91 amplitudes (74 for TNOs, and 17 for Centaurs) from Table 1. We note that some papers only report the amplitude, or some restriction in the amplitude, but no period. That is the reason why the number of amplitude determinations differ from the rotational period determinations.

The first noticeable characteristic in that plot is that nearly $70 \%$ of the objects (in both cases, TNOs and Centaurs) have light-curve amplitudes smaller than $0.2 \mathrm{mag}$. The reasons for observing a flat light curve could be a spherical body with low contrast albedo marks on the surface, or a pole-on configuration, that is, a rotational axis oriented toward the observer. This last option is the least probable of all, so it is reasonable to say that most of the observed TNOs are "large" round objects with no significant 
Table 1. List of TNOs whose variability has been studied. When a multiple determination of the rotational period is present, the preferred period is marked in bold.

\begin{tabular}{|c|c|c|c|c|c|c|}
\hline Object & Designation & $\begin{array}{c}\text { Single Peak } \\
\text { period [h] }\end{array}$ & $\begin{array}{c}\text { Double Peak } \\
\text { period }[\mathrm{h}]\end{array}$ & $\begin{array}{l}\text { Amplitude } \\
\text { [mag] }\end{array}$ & $\begin{array}{c}\text { Absolute } \\
\text { magnitude }^{a}\end{array}$ & Ref. $^{b}$ \\
\hline (134340) Pluto & & 153.6 & - & 0.33 & -0.7 & B97,TH90 \\
\hline Charon & & 153.6 & - & 0.08 & 0.9 & B97 \\
\hline (148780) Altjira & $2001 \mathrm{UQ}_{18}$ & - & - & $<0.10$ & 5.6 & S07 \\
\hline (55576) Amycus & $2002 \mathrm{~GB}_{10}$ & 9.76 & 19.52 & $0.16 \pm 0.01$ & 7.8 & Th09 \\
\hline \multirow[t]{2}{*}{ (8405) Asbolus } & 1995 GO & 4.47 & $8.9351 \pm 0.003$ & 0.55 & 9.0 & D98a,K00 \\
\hline & & - & - & 0.34 & $\ldots$ & RT99 \\
\hline \multirow[t]{2}{*}{ (54598) Bienor } & $2000 \mathrm{QC}_{243}$ & $4.57 \pm 0.02$ & $9.14 \pm 0.04$ & $0.75 \pm 0.09$ & 7.6 & $\mathrm{O} 03 \mathrm{~b}$ \\
\hline & & 9.17 & - & $0.34 \pm 0.08$ & $\ldots$ & $\mathrm{R} 07 \mathrm{a}$ \\
\hline (66652) Borasisi & $1999 \mathrm{RZ}_{253}$ & - & - & $<0.05$ & 5.9 & LL06 \\
\hline (65489) Ceto & $2003 \mathrm{FX}_{128}$ & - & $4.43 \pm 0.03$ & $0.13 \pm 0.02$ & 6.3 & SJ02 \\
\hline (19521) Chaos & $1998 \mathrm{WH}_{24}$ & - & - & $<0.10$ & 4.9 & SJ02,LL06 \\
\hline (10199) Chariklo & $1997 \mathrm{CU}_{26}$ & - & - & $<0.1$ & 6.4 & LL06 \\
\hline (2060) Chiron & 1977 UB & - & $5.9180 \pm 0.0001$ & $0.088 \pm 0.003$ & 6.5 & B89 \\
\hline \multirow[t]{2}{*}{ (83982) Crantor } & $2002 \mathrm{GO}_{9}$ & $(6.97$ or 9.67$) \pm 0.04$ & - & $0.14 \pm 0.04$ & 9.1 & $\mathrm{O} 03 \mathrm{~b}$ \\
\hline & & - & - & 0.34 & $\ldots$ & RT99 \\
\hline (60558) Echeclus & $2000 \mathrm{EC}_{98}$ & 13.401 & 26.802 & $0.24 \pm 0.06$ & 9.0 & R03 \\
\hline \multirow[t]{2}{*}{ (31824) Elatus } & $1999 \mathrm{UG}_{5}$ & 13.25 & - & 0.24 & 10.1 & G01 \\
\hline & & $13.41 \pm 0.04$ & - & $0.102 \pm 0.005$ & $\ldots$ & B02 \\
\hline \multirow[t]{4}{*}{ (136199) Eris } & $2003 \mathrm{UB}_{313}$ & $13.69 / 28.08 / 32.13$ & - & $<0.1 \pm 0.01$ & -1.2 & D07 \\
\hline & & $3.55 ?$ & - & $\sim 0.05$ & $\ldots$ & L07 \\
\hline & & - & - & $<0.01$ & $\ldots$ & $\mathrm{R} 07 \mathrm{a}, \mathrm{S} 07$ \\
\hline & & 25.92 & - & 0.1 & $\ldots$ & R08 \\
\hline \multirow[t]{3}{*}{ (136108) Haumea } & $2003 \mathrm{EL}_{61}$ & - & $3.9154 \pm 0.0002$ & $0.28 \pm 0.04$ & 0.2 & R06 \\
\hline & & - & $3.9155 \pm 0.0001$ & $0.29 \pm 0.02$ & $\ldots$ & LJ08 \\
\hline & & 1.96 & 3.92 & $0.23 \pm 0.02$ & $\ldots$ & Th09 \\
\hline (10370) Hylonome & $1995 \mathrm{DW}_{2}$ & - & - & $<0.04$ & 8.0 & RT99 \\
\hline \multirow[t]{3}{*}{ (38628) Huya } & $2000 \mathrm{~EB}_{173}$ & $6.68 / \mathbf{6 . 7 5} / 6.82$ & - & $<0.1$ & 4.7 & $\mathrm{O} 03 \mathrm{~b}$ \\
\hline & & - & - & $<0.06$ & $\ldots$ & $\mathrm{O} 03 \mathrm{~b}, \mathrm{SJ} 02, \mathrm{~S} 02$ \\
\hline & & - & - & $<0.04$ & $\ldots$ & SJ03,LL06 \\
\hline \multirow[t]{2}{*}{ (28978) Ixion } & $2001 \mathrm{KX}_{76}$ & - & - & $<0.05$ & 3.2 & O03b,SJ03 \\
\hline & & - & - & $<0.10$ & $\ldots$ & SJ02,LL06 \\
\hline \multirow[t]{2}{*}{ (136472) Makemake } & $2005 \mathrm{FY}_{9}$ & $11.24 \pm 0.01$ & $20.54 / 22.48$ & $0.03 \pm 0.01$ & -0.3 & $\mathrm{O} 07$ \\
\hline & & 7.65 & 15.30 & $0.014 \pm 0.002$ & $\ldots$ & Th09 \\
\hline \multirow[t]{2}{*}{ (7066) Nessus } & $1993 \mathrm{HA}_{2}$ & - & - & 0.5 & 9.6 & RT99 \\
\hline & & - & - & $<0.2$ & $\ldots$ & D98b \\
\hline (52872) Okyrhoe & $1998 \mathrm{SG}_{35}$ & $4.86 / 6.08$ & $9.72 / 12.16$ & $0.07 \pm 0.01$ & 11.3 & Th09 \\
\hline \multirow[t]{4}{*}{ (90482) Orcus } & 2004 DW & $7.09 / \mathbf{1 0 . 4 7} \pm \mathbf{0 . 0 1} / 17.43$ & 20.94 & $0.03 \pm 0.01$ & 2.3 & O06 \\
\hline & & 13.19 & - & 0.18 & $\ldots$ & $\mathrm{RO} 7 \mathrm{a}$ \\
\hline & & - & - & $<0.03$ & $\ldots$ & S07 \\
\hline & & 10.47 & 20.94 & $0.03 \pm 0.01$ & $\ldots$ & Th09 \\
\hline \multirow[t]{2}{*}{ (5145) Pholus } & $1992 \mathrm{AD}$ & - & 9.98 & $0.15 / 0.60$ & 7.0 & B92,H92,F01,T05 \\
\hline & & - & - & 0.15 & $\ldots$ & RT99 \\
\hline \multirow[t]{4}{*}{ (50000) Quaoar } & $2002 \mathrm{LM}_{60}$ & - & $17.6788 \pm 0.0004$ & $0.13 \pm 0.03$ & 2.6 & $\mathrm{O} 03 \mathrm{~b}$ \\
\hline & & 8.84 & - & $0.18 \pm 0.10$ & $\ldots$ & $\mathrm{R} 07 \mathrm{a}$ \\
\hline & & 9.42 & 18.84 & $\sim 0.3$ & $\ldots$ & L07 \\
\hline & & 8.84 & 17.68 & $0.13 \pm 0.04$ & $\ldots$ & Th09 \\
\hline (90377) Sedna & $2003 \mathrm{VB}_{12}$ & $10.273 \pm 0.003$ & - & 0.02 & 1.6 & G05 \\
\hline (88611) Teharonhiawako & $2001 \mathrm{QT}_{297}$ & - & - & $<0.15$ & 5.5 & Os03 \\
\hline (32532) Thereus & $2001 \mathrm{PT}_{13}$ & $4.1546 \pm 0.0001$ & $8.3091 \pm 0.0001$ & $0.16 \pm 0.02$ & 9.0 & $\mathrm{O} 03 \mathrm{~b}$ \\
\hline & & - & 8.34 & 0.16 & $\ldots$ & FD03 \\
\hline & & - & 8.34 & $0.34 \pm 0.08$ & $\ldots$ & $\mathrm{R} 07 \mathrm{a}$ \\
\hline & & - & 8.4 & 0.15 & $\ldots$ & F01 \\
\hline (42355) Typhon & $2002 \mathrm{CR}_{46}$ & 9.67 & 19.34 & $0.06 \pm 0.01$ & 7.2 & $\mathrm{O} 03 \mathrm{~b}$ \\
\hline & & - & - & $<0.05$ & $\ldots$ & SJ03 \\
\hline & & $>5$ & - & - & $\ldots$ & D08 \\
\hline & & 9.67 & 19.34 & $0.06 \pm 0.01$ & $\ldots$ & Th09 \\
\hline (20000) Varuna & $2000 \mathrm{WR}_{106}$ & 3.1718 & $6.3436 \pm 0.0001$ & $0.43 \pm 0.01$ & 3.6 & $\mathrm{O} 03 \mathrm{~b}$ \\
\hline & & - & 6.34 & $0.42 \pm 0.03$ & $\ldots$ & SJ02 \\
\hline & & 3.17 & 6.34 & 0.5 & $\ldots$ & F01 \\
\hline & & - & $6.34358 \pm 0.00002$ & 0.42 & $\ldots$ & B06 \\
\hline & & - & 6.344 & $0.49 \pm 0.17$ & $\ldots$ & $\mathrm{R} 07 \mathrm{a}$ \\
\hline & & 3.1709 & 6.3418 & $0.43 \pm 0.01$ & $\ldots$ & Th09 \\
\hline (15789) & $1993 \mathrm{SC}$ & 7.7 & - & 0.04 & 6.9 & T97 \\
\hline & & - & - & $<0.04$ & $\ldots$ & RT99 \\
\hline
\end{tabular}


Table 1. continued.

\begin{tabular}{|c|c|c|c|c|c|c|}
\hline Object & Designation & $\begin{array}{c}\text { Single Peak } \\
\text { period }[\mathrm{h}]\end{array}$ & $\begin{array}{c}\text { Double Peak } \\
\text { period }[\mathrm{h}]\end{array}$ & $\begin{array}{l}\text { Amplitude } \\
\text { [mag] }\end{array}$ & $\begin{array}{l}\text { Absolute } \\
\text { magnitude }^{a}\end{array}$ & Ref. $^{b}$ \\
\hline \multirow[t]{2}{*}{$(15820)$} & $1994 \mathrm{~TB}$ & $3.0 / 3.5$ & $6.0 / 7.0$ & $0.26 / 0.34$ & 7.1 & RT99 \\
\hline & & - & - & $<0.04$ & $\ldots$ & SJ02 \\
\hline \multirow[t]{2}{*}{ (19255) } & $1994 \mathrm{VK}_{8}$ & $3.9 / 4.3 / 4.7 / 5.2$ & 7.8/8.6/9.4/10.4 & 0.42 & 7.0 & RT99 \\
\hline & & 4.75 & - & - & $\ldots$ & CB99 \\
\hline (24835) & $1995 \mathrm{SM}_{55}$ & $4.04 \pm 0.03$ & $8.08 \pm 0.03$ & $0.19 \pm 0.05$ & 4.8 & SJ02 \\
\hline \multirow[t]{3}{*}{ (32929) } & $1995 \mathrm{QY}_{9}$ & 3.5 & 7.0 & 0.60 & 7.5 & RT99 \\
\hline & & Between 3.3 and 3.7 & - & 0.60 & $\ldots$ & RT99 \\
\hline & & - & $7.3 \pm 0.1$ & $0.60 \pm 0.04$ & $\ldots$ & RT99,SJ02 \\
\hline (26181) & $1996 \mathrm{GQ}_{21}$ & - & - & $<0.10$ & 5.2 & SJ02 \\
\hline \multirow[t]{3}{*}{ (15874) } & $1996 \mathrm{TL}_{66}$ & 12.1 & - & $<0.12$ & 5.4 & O06 \\
\hline & & - & - & $<0.06$ & $\ldots$ & LJ98,RT99 \\
\hline & & 6/8.04/ 12 & 24 & $0.09 \pm 0.02$ & $\ldots$ & Th09 \\
\hline \multirow[t]{4}{*}{ (19308) } & $1996 \mathrm{TO}_{66}$ & $3.96 \pm 0.04$ & $\mathbf{7 . 9 2} \pm \mathbf{0 . 0 4} / 5.9 / 9.6$ & $0.25 \pm 0.05$ & 4.5 & SJ03 \\
\hline & & - & 11.9 & $0.25 \pm 0.05$ & $\ldots$ & B06 \\
\hline & & - & $6.25 \pm 0.03$ & 0.12 to 0.33 & $\ldots$ & $\mathrm{H} 0 \mathrm{O}$ \\
\hline & & - & - & $<0.10$ & $\ldots$ & RT99 \\
\hline \multirow[t]{2}{*}{ (15875) } & $1996 \mathrm{TP}_{66}$ & 1.96 & - & $<0.04$ & 6.8 & CB99 \\
\hline & & - & - & $<0.12$ & $\ldots$ & RT99 \\
\hline \multirow[t]{3}{*}{ (118228) } & $1996 \mathrm{TQ}_{66}$ & - & - & $<0.22$ & 7.1 & RT99 \\
\hline & $1996 \mathrm{TS}_{66}$ & - & - & $<0.14$ & 6.5 & LL06 \\
\hline & & - & - & $<0.16$ & $\ldots$ & RT99 \\
\hline \multirow[t]{3}{*}{ (79360) } & $1997 \mathrm{CS}_{29}$ & - & - & $<0.08$ & 5.1 & SJ02 \\
\hline & & - & - & $<0.22$ & $\ldots$ & RT99 \\
\hline & $1997 \mathrm{CV}_{29}$ & - & 15.8 & 0.4 & 7.3 & CK04 \\
\hline (33128) & $1998 \mathrm{BU}_{48}$ & $(4.9$ or 6.3$) \pm 0.1$ & $(9.8$ or 12.6$) \pm 0.1$ & $0.68 \pm 0.04$ & 7.2 & SJ02 \\
\hline (91133) & $1998 \mathrm{HK}_{151}$ & - & - & $<0.15$ & 7.6 & SJ02 \\
\hline \multirow[t]{2}{*}{ (26308) } & $1998 \mathrm{SM}_{165}$ & - & $7.1 \pm 0.1$ & $0.45 \pm 0.03$ & 5.8 & SJ02 \\
\hline & & 3.983 & - & 0.56 & $\ldots$ & $\mathrm{R} 01$ \\
\hline \multirow[t]{2}{*}{$(35671)$} & $1998 \mathrm{SN}_{165}$ & - & 8.84 & $0.16 \pm 0.01$ & 5.8 & LL06 \\
\hline & & 5.03 & - & 0.15 & $\ldots$ & P02 \\
\hline \multirow[t]{3}{*}{ (33340) } & $1998 \mathrm{VG}_{44}$ & - & - & $<0.10$ & 6.5 & SJ02 \\
\hline & $1998 X_{95}$ & - & - & $<0.1$ & 6.2 & CB01 \\
\hline & & 1.31 & - & $\sim 0.1$ & $\ldots$ & CB01 \\
\hline (26375) & $1999 \mathrm{DE}_{9}$ & $>12 ?$ & - & $<0.10$ & 4.7 & SJ02 \\
\hline (79983) & $1999 \mathrm{DF}_{9}$ & 3.3 & $6.65 / 9.2$ & $0.40 \pm 0.02$ & 6.1 & LL06 \\
\hline (40314) & $1999 \mathrm{KR}_{16}$ & $(5.840$ or 5.929$) \pm 0.001$ & $(11.680$ or 11.858$) \pm 0.002$ & $0.18 \pm 0.04$ & 5.8 & $\mathrm{SJ} 02$ \\
\hline \multirow[t]{3}{*}{ (47171) } & $1999 \mathrm{TC}_{36}$ & 6.21 & - & 0.06 & 4.9 & $\mathrm{O} 03 \mathrm{~b}$ \\
\hline & & - & - & $<0.07$ & $\ldots$ & LL06 \\
\hline & & - & - & $<0.05$ & $\ldots$ & SJ03 \\
\hline \multirow[t]{8}{*}{ (29981) } & $1999 \mathrm{TD}_{10}$ & $7.71 \pm 0.02$ & - & $0.65 \pm 0.05$ & 8.8 & $\mathrm{O} 03 \mathrm{~b}$ \\
\hline & & - & 15.45 & 0.65 & $\ldots$ & $\mathrm{R} 03, \mathrm{C} 03$ \\
\hline & & - & 15.382 & $0.41 \pm 0.08$ & $\ldots$ & M04 \\
\hline & & - & 15.3833 & $0.53 \pm 0.03$ & $\ldots$ & RO5a \\
\hline & & 5.8 & - & $0.65 \pm 0.05$ & $\ldots$ & $\mathrm{C} 00$ \\
\hline & $1999 \mathrm{TZ}_{1}$ & - & 10.438 & $<0.1$ & 9.3 & M08 \\
\hline & & 5 & - & $0.15 \pm 0.02$ & $\ldots$ & D08 \\
\hline & & 5.211 & 10.422 & $0.06 \pm 0.01$ & $\ldots$ & Th09 \\
\hline (80806) & $2000 \mathrm{CM}_{105}$ & - & - & $<0.14$ & 6.3 & LL06 \\
\hline & $2000 \mathrm{CP}_{104}$ & - & - & 0.06 & 6.7 & R08 \\
\hline & $2000 \mathrm{FV}_{53}$ & 3.79 & - & $0.07 \pm 0.02$ & 8.2 & ТВ05 \\
\hline & & - & 7.5 & 0.07 & $\ldots$ & TB05 \\
\hline & $2000 \mathrm{CP}_{104}$ & - & - & 0.06 & 6.7 & R08 \\
\hline (47932) & $2000 \mathrm{GN}_{171}$ & - & $8.329 \pm 0.05$ & $0.61 \pm 0.03$ & 6.0 & SJ02 \\
\hline & & - & $8.329 \pm 0.05$ & $0.60 \pm 0.03$ & $\ldots$ & D08 \\
\hline (82075) & $2000 \mathrm{YW}_{134}$ & - & - & $<0.10$ & 5.0 & SJ03 \\
\hline (150642) & $2001 \mathrm{CZ}_{31}$ & - & $4.71 / 5.23$ & $0.21 \pm 0.02$ & 5.7 & LL06 \\
\hline & & - & - & $<0.20$ & $\ldots$ & SJ02 \\
\hline (82158) & $2001 \mathrm{FP}_{185}$ & - & - & $<0.06$ & 6.1 & SJ03 \\
\hline (82155) & $2001 \mathrm{FZ}_{173}$ & - & - & $<0.06$ & 6.2 & SJ02 \\
\hline & $2001 \mathrm{KD}_{77}$ & - & - & $<0.07$ & 5.8 & SJ03 \\
\hline & $2001 \mathrm{QF}_{298}$ & - & - & $<0.12$ & 4.7 & SJ03 \\
\hline (139775) & $2001 \mathrm{QG}_{298}$ & $6.89 \pm 0.0002$ & $13.7744 \pm 0.0004$ & $1.14 \pm 0.04$ & 7.0 & SJ04 \\
\hline (88611B) & $2001 \mathrm{QT}_{297 B}$ & 4.75 & - & 0.6 & 5.5 & Os03 \\
\hline & & $5.50 \pm 0.02$ & - & - & $\ldots$ & K06 \\
\hline (42301) & $2001 \mathrm{UR}_{163}$ & - & - & $<0.08$ & 4.2 & SJ03 \\
\hline
\end{tabular}


Table 1. continued.

\begin{tabular}{|c|c|c|c|c|c|c|}
\hline Object & Designation & $\begin{array}{c}\text { Single Peak } \\
\text { period }[\mathrm{h}]\end{array}$ & $\begin{array}{c}\text { Double Peak } \\
\text { period }[\mathrm{h}]\end{array}$ & $\begin{array}{c}\text { Amplitude } \\
{[\mathrm{mag}]}\end{array}$ & $\begin{array}{l}\text { Absolute } \\
\text { magnitude }\end{array}$ & Ref. $^{b}$ \\
\hline \multirow[t]{3}{*}{$(126154)$} & $2001 \mathrm{YH}_{140}$ & $6.22 / \mathbf{8 . 4 5} \pm \mathbf{0 . 0 5} / 12.99$ & $12.38 / \mathbf{1 6 . 8 0} / 26.72$ & $0.16 \pm 0.05$ & 5.4 & 006 \\
\hline & & - & 13.25 & $0.21 \pm 0.04$ & $\ldots$ & S07 \\
\hline & & 6.19/8.40/13.36 & $12.38 / 16.80 / \mathbf{2 6 . 7 2}$ & $0.16 \pm 0.05$ & $\ldots$ & Th09 \\
\hline \multirow[t]{2}{*}{ (55565) } & $2002 \mathrm{AW}_{197}$ & $6.49 / \mathbf{8 . 8 7} \pm \mathbf{0 . 0 1} / 13.94 / 15.82$ & 17.74 & $0.04 \pm 0.01$ & 3.3 & O06 \\
\hline & & 8.78 & 17.56 & $0.04 \pm 0.01$ & $\ldots$ & Th09 \\
\hline \multirow[t]{2}{*}{ (95626) } & $2002 \mathrm{GP}_{32}$ & $\sim 3.3$ & $\sim 6.6$ & $>1$ & 6.7 & K06 \\
\hline & & - & - & $<0.03$ & $\ldots$ & S07 \\
\hline (95626) & $2002 \mathrm{GZ}_{32}$ & - & $5.80 \pm 0.03$ & $0.15 \pm 0.03$ & 6.8 & D08 \\
\hline (73480) & $2002 \mathrm{PN}_{34}$ & $(4.23$ or 5.11$) \pm 0.03$ & $(8.45$ or 10.22$) \pm 0.06$ & $0.18 \pm 0.04$ & 8.2 & O03b \\
\hline \multirow[t]{3}{*}{ (55636) } & $2002 \mathrm{TX}_{300}$ & $(8.12$ or 12.10$) \pm 0.08$ & $(\mathbf{1 6 . 2 4}$ or 24.20$) \pm 0.08$ & $0.08 \pm 0.02$ & 3.3 & SJ03 \\
\hline & & $7.89 \pm 0.03$ & 15.78 & $0.09 \pm 0.08$ & $\ldots$ & $\mathrm{O} 04$ \\
\hline & & 4.08 & 8.16 & $0.04 \pm 0.01$ & $\ldots$ & Th09 \\
\hline \multirow[t]{3}{*}{ (55637) } & $2002 \mathrm{UX}_{25}$ & 7.19 or 8.4 & 14.38 & $0.21 \pm 0.06$ & 3.6 & R05b \\
\hline & & - & 16.782 & $0.13 \pm 0.09$ & $\ldots$ & RO5a \\
\hline & & - & - & $<0.06$ & $\ldots$ & SJ03 \\
\hline \multirow[t]{3}{*}{$(555638)$} & $2002 \mathrm{VE}_{95}$ & $6.76 / 6.88 / 7.36 / 9.47$ & - & $0.08 \pm 0.04$ & 5.3 & O06 \\
\hline & & - & - & $<0.06$ & $\ldots$ & SJ03 \\
\hline & & $4.90 / 6.18 / \mathbf{9 . 9 7 / 1 7 . 2 3}$ & $9.80 / 12.36 / \mathbf{1 9 . 9 4 / 3 4 . 4 6 ~}$ & $0.04 \pm 0.01$ & $\ldots$ & Th09 \\
\hline \multirow[t]{2}{*}{ (136204) } & $2003 \mathrm{WL}_{7}$ & 8.24 & 16.48 & $0.04 \pm 0.01$ & 8.7 & Th09 \\
\hline & $2005 \mathrm{CB}_{79}$ & 6.76 & 13.52 & $0.13 \pm 0.02$ & 5.0 & Th09 \\
\hline (145451) & $2005 \mathrm{RM}_{43}$ & 6.71 & 13.42 & $0.04 \pm 0.01$ & 4.4 & Th09 \\
\hline (145452) & $2005 \mathrm{RN}_{43}$ & 5.62 or 7.32 & 11.24 or 14.64 & $0.04 \pm 0.01$ & 3.9 & Th09 \\
\hline (145453) & $2005 \mathrm{RR}_{43}$ & 7.87 & 15.74 & $0.06 \pm 0.01$ & 4.0 & Th09 \\
\hline \multirow[t]{2}{*}{ (145486) } & $2005 \mathrm{UJ}_{438}$ & 4.16 & 8.32 & $0.11 \pm 0.01$ & 10.5 & Th09 \\
\hline & $2007 \mathrm{UL}_{126}$ or $2002 \mathrm{KY}_{14}$ & 3.56 or 4.20 & 7.12 or 8.40 & $0.10 \pm 0.01$ & 9.4 & Th09 \\
\hline (120347) & $2004 \mathrm{SB}_{60}$ & 6.09 or 8.01 & 12.18 or 16.02 & $0.03 \pm 0.01$ & 4.4 & Th09 \\
\hline (144897) & $2004 \mathrm{UX}_{10}$ & 5.68 & 11.36 & $0.08 \pm 0.01$ & 4.7 & Th09 \\
\hline \multirow[t]{4}{*}{ (119979) } & $2002 \mathrm{WC}_{19}$ & - & - & $<0.03$ & 5.1 & S07 \\
\hline & $2003 \mathrm{AZ}_{84}$ & $4.32 / 5.28 / 6.72 / 6.76 \pm \mathbf{0 . 0 1}$ & - & $0.14 \pm 0.03$ & 3.6 & O06 \\
\hline & & - & $5.72 \pm 0.05$ & $0.14 \pm 0.03$ & $\ldots$ & SJ02 \\
\hline & & 6.79 & 13.58 & $0.10 \pm 0.01$ & $\ldots$ & Th09 \\
\hline \multirow[t]{5}{*}{ (120061) } & $2003 \mathrm{CO}_{1}$ & 4.51 & 9.02 & $0.06 \pm 0.01$ & 8.9 & Th09 \\
\hline & $2003 \mathrm{BF}_{91}$ & 9.1/7.3 & - & $1.09 \pm 0.25$ & 11.7 & ТВ05 \\
\hline & $2003 \mathrm{BG}_{91}$ & 4.2/4.5/4.6/4.9 & - & $0.18 \pm 0.75$ & 10.7 & ТВ05 \\
\hline & $2003 \mathrm{BH}_{91}$ & - & - & 0.42 & 11.9 & ТВ05 \\
\hline & & 2.8 & - & $<0.15$ & $\ldots$ & ТВ05 \\
\hline \multirow[t]{3}{*}{ (120132) } & $2003 \mathrm{FY}_{128}$ & - & - & $<0.08$ & 5.0 & S07 \\
\hline & & $>7$ & - & - & $\ldots$ & D08 \\
\hline & & 8.54 & 17.08 & $0.12 \pm 0.01$ & 5.0 & Th09 \\
\hline \multirow[t]{6}{*}{ (120178) } & $2003 \mathrm{OP}_{32}$ & $4.845 \pm 0.03$ & - & $0.26 \pm 0.04$ & 4.1 & $\mathrm{Ra} 08$ \\
\hline & & 4.05 & 8.10 & $0.10 \pm 0.01$ & $\ldots$ & Th09 \\
\hline & $2003 \mathrm{QB}_{112}$ & - & - & 0.46 & 7.0 & R08 \\
\hline & $2003 \mathrm{QY}_{90 \mathrm{~A}}$ & $3.4 \pm 1.1$ & - & $0.34 \pm 0.06$ & 6.3 & KO6а \\
\hline & $2003 \mathrm{QY}_{90 B}$ & $7.1 \pm 2.9$ & - & $0.90 \pm 0.18$ & 6.3 & K06a \\
\hline & 2003 QY $_{111}$ & - & - & 0.60 & 6.6 & R08 \\
\hline (174567) & $2003 \mathrm{MW}_{12}$ & $5.90 / 7.87$ & $11.80 / 15.74$ & $0.06 \pm 0.01$ & 3.6 & Th09 \\
\hline \multirow[t]{2}{*}{ (84922) } & $2003 \mathrm{VS}_{2}$ & 3.71 or 4.39 & 7.42 & $0.17 \pm 0.01$ & 4.2 & O06,Th09 \\
\hline & & - & $7.41 \pm 0.01$ & $0.21 \pm 0.02$ & $\ldots$ & S07 \\
\hline \multirow[t]{2}{*}{ (90568) } & $2004 \mathrm{GV}_{9}$ & - & - & $<0.1$ & 4.0 & S07 \\
\hline & & - & $5.86 \pm 0.03$ & $0.16 \pm 0.03$ & $\ldots$ & D08 \\
\hline (120348) & $2004 \mathrm{TY}_{364}$ & $5.85 \pm 0.01$ & $11.7 \pm 0.01$ & $0.22 \pm 0.02$ & 4.5 & S07 \\
\hline
\end{tabular}

${ }^{a}$ MPC values. ${ }^{b}$ References: B97: Buie et al. (1997); D98a: Davies et al. (1998a); K00: Kern et al. (2000); RT99: Romanishin \& Tegler (1999); O03b: Ortiz et al. (2003a); R07a: Rabinowitz et al. (2007); LL06: Lacerda \& Luu (2006); D08: Dotto et al. (2008); SJ02: Sheppard \& Jewitt (2002); B89: Bus et al. (1989); R03: Rousselot et al. (2003); G01: Gutierrez et al. (2001); B02: Bauer et al. (2002); D07: Duffard et al. (2008); L07: Lin et al. (2007); S07: Sheppard (2007); R08: Roe et al. (2008); S02: Schaefer \& Rabinowitz (2002); SJ03: Sheppard \& Jewitt (2003); B03: Bauer et al. (2003); O06: Ortiz et al. (2006); B92: Buie \& Bus (1992); F01: Farnham (2001); T05: Tegler et al. (2005); H92: Hoffmann et al. (1992); O03a: Ortiz et al. (2003b); G05: Gaudi et al. (2005); Os03: Osip et al. (2003); FD03: Farnham \& Davies (2003); B06: Belskaya et al. (2006); T97: Tegler et al. (1997); CB99: Collander-Brown et al. (1999); LJ98: Luu \& Jewitt (1998); H00: Hainaut et al. (2000); CK04: Chorney \& Kavelaars (2004); R01: Romanishin et al. (2001); P02: Peixinho et al. (2002); CB01: Collander-Brown et al. (2001); C03: Choi et al. (2003); M04: Mueller et al. (2004); R05a: Rousselot et al. (2005a); C00: Consolmagno et al. (2000); M08: Moullet et al. (2008); TB05: Trilling \& Bernstein (2006); R06: Rabinowitz et al. (2006); SJ04: Sheppard \& Jewitt (2004); K06b: Kern (2006); O04: Ortiz et al. (2004); R05b: Rousselot et al. (2005b); LJ08: Lacerda et al. (2008); Ra08: Rabinowitz et al. (2008); K06a: Kern \& Elliot (2006); O07: Ortiz et al. (2007); D98b: Davies et al. (1998b); D01: Davies et al. (2001); TH90: Tholen \& Buie (1990); Th09: Thirouin et al. (2009). 


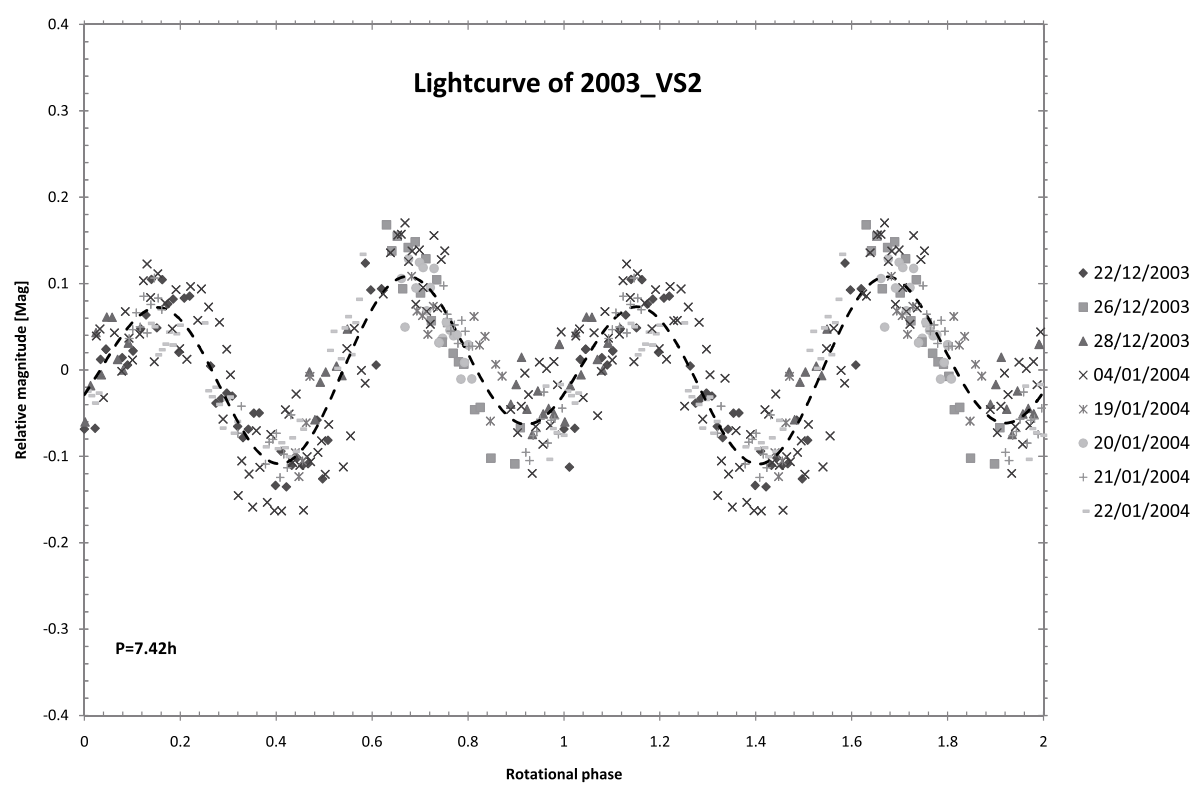

Fig. 1. Example of a KBO light curve from Thirouin et al. (2009) obtained during several nights.

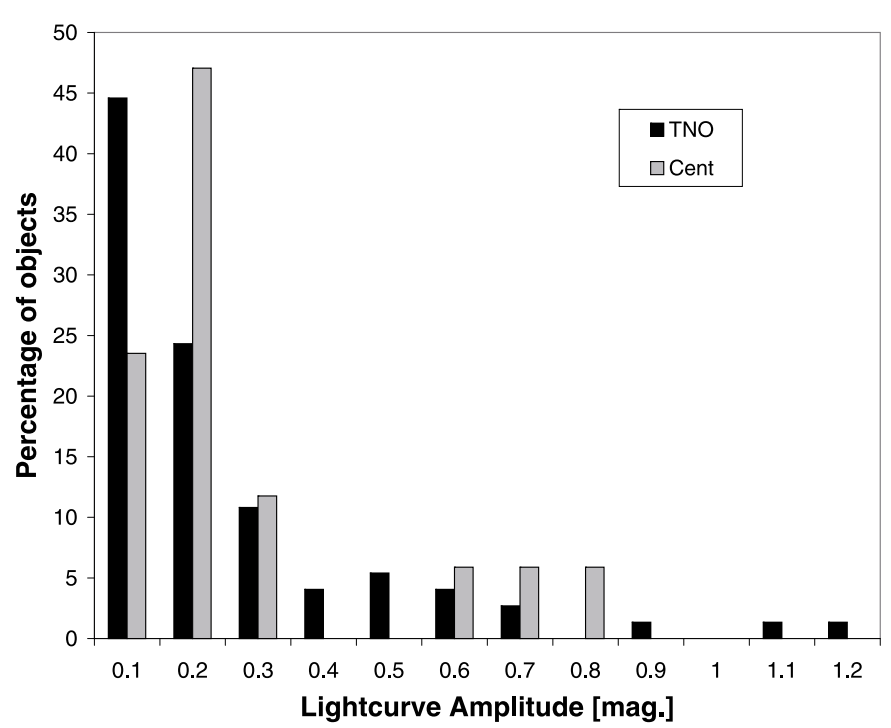

Fig. 2. Percentage of objects vs. peak-to-peak light curve amplitude for all TNOs (black) and Centaurs (grey).

inhomogeneities on their surfaces. Objects with flat light curves correspond in general to a less collisionally evolved population. Of course, the large abundance of the low light-curve amplitude population could be to some degree an observational bias because the KBOs with known rotational properties are mainly the brightest ones. For the Centaurs, the vast majority of objects have light-curve amplitudes between $0.1 \mathrm{mag}$ and $0.2 \mathrm{mag}$, which is indicative of some elongation in the shape of the bodies.

\subsection{Period distribution}

In the following analysis, all objects with known rotational period are considered. An exception was made for Pluto, which was excluded. This exception is because the long rotational period (due to the tidal locking of the system) cannot be representative of the rotational properties of the sample.

From KBO light curves alone it is difficult or even impossible to determine whether the variability is caused by either albedo variations or an elongated shape. Therefore, the rotation

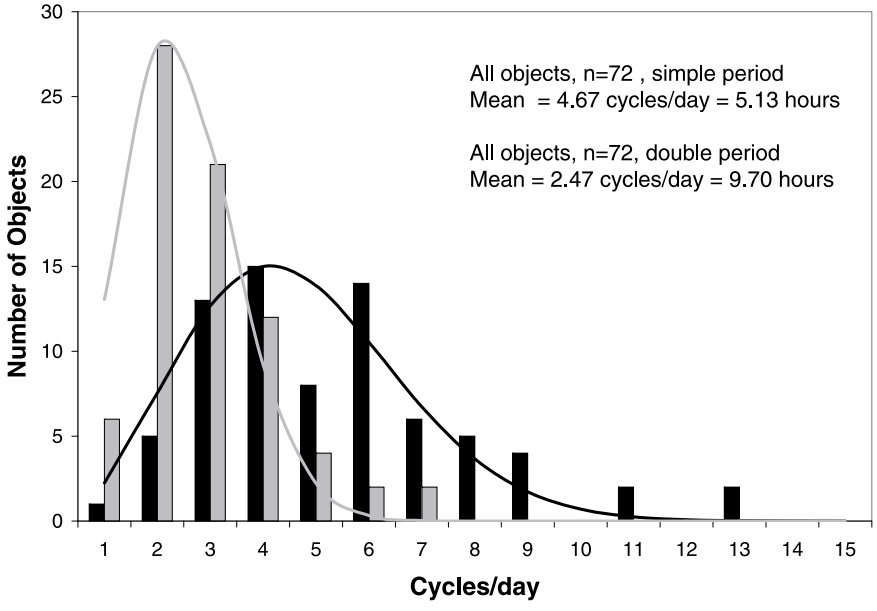

Fig. 3. Histogram in rotational frequencies for all the objects considering only a simple period rotation (black) and double period rotation (grey). A maxwellian fit to the single peak period for all the object gives a mean not-reliable lower period of $5.13 \mathrm{~h}$ (black bars/line). Double peak period is also represented (grey bars). The maxwellian fit to the data gives a mean not-reliable upper period of $9.70 \mathrm{~h}$.

periods derived from the photometric periods often have an uncertainty of a factor 2. A double-peaked periodic light curve is expected to be seen in a non-spherical body, since the projected cross-section should have two minima (short axis) and two maxima (long axis) during one complete rotation of the KBO. Light curves caused by surface inhomogeneities are single peaked. Figure 3 (in black) shows the histogram of rotational frequencies (single-peak rotational period) for all the objects found in the literature. This histogram was compiled by assuming that all objects have single-peaked light curves, which is obviously not true and is an extreme case. For the well known cases in which the light curves are certainly double-peaked, we used the rotation period corresponding to half the true rotation period. Therefore, the resulting distribution of rotation periods has a short mean value, which is a crude lower limit to the mean rotation period. A Maxwellian curve was fitted and overplotted in the histogram for a mean period of $5.13 \mathrm{~h}$. 

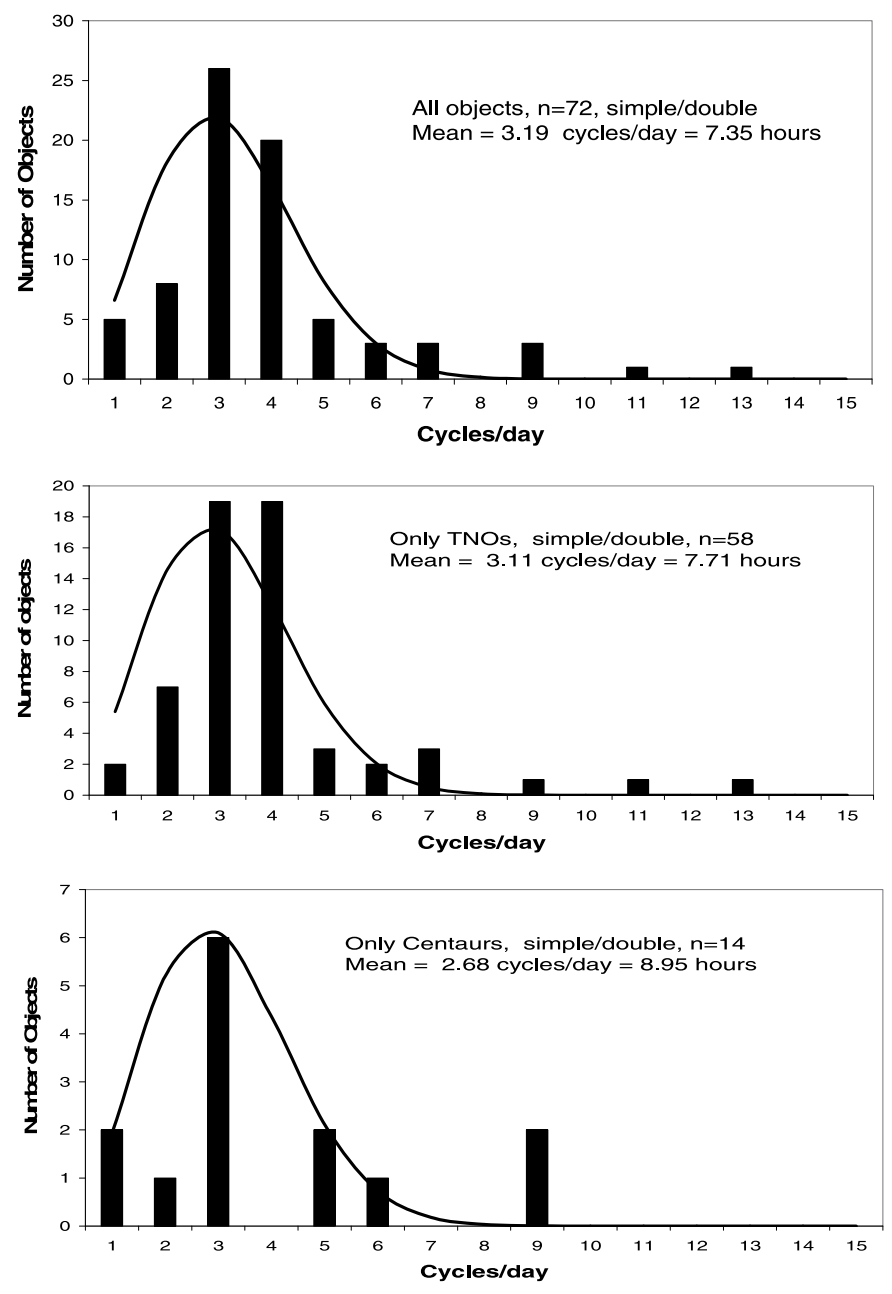

Fig. 4. Histogram in cycles/day for the whole sample (top panel), the TNOs (center panel) and Centaurs (lower panel). From maxwellian fits to the rotational frequencies distribution the mean rotation rates are $7.35 \mathrm{~h}$ for the entire sample, $7.71 \mathrm{~h}$ for the TNOs alone and $8.95 \mathrm{~h}$ for the Centaurs. We chose to use the single peak period for the objects whose amplitude is $<=0.15$ mag and double peak period for the objects whose amplitude is $>0.15 \mathrm{mag}$.

The obvious next exercise is to plot the histogram of rotational frequencies for all objects with double-peak light curves (in other words, we assume that all rotation periods are twice the periods derived by a basic periodogram analysis). This is again an unrealistic result, but provides an upper limit to the rotational period distribution. This is the same as considering all the light curves to be produced by non-spherical bodies. The Maxwellian fit infers a mean frequency of 2.47 cycles/day or $9.70 \mathrm{~h}$ (data in grey).

Finally, a more reliable histogram (presented in Fig. 4) was compiled by assuming that all the light curves with amplitudes smaller than or equal to $0.15 \mathrm{mag}$ are single peaked (this is equivalent to assuming that the main cause for their rotational variation is albedo markings) and those with amplitudes larger than 0.15 mag are double peaked. Thus, with this criterium we remove the factor of 2 ambiguity and derive a more realistic distribution. There is also the possibility of deciding on a case-bycase basis, but some cases are ambiguous.

If we calculate the raw average rotational period, the obtained values from Table 1 are $6.95,6.88$, and $6.75 \mathrm{~h}$ for the entire sample, for both the TNOs and the Centaurs only,
Table 2. Most important correlations found using the light curve parameters and orbital/physical variables as described in the text. The parameters presented are the light curve amplitude (Ampl.), the perihelion $(q)$ and aphelion $(Q)$ distances, inclination $(i)$, eccentricity $(e)$ and absolute magnitude $(H)$.

\begin{tabular}{llccc}
\hline \hline Population & Correlated Magnitudes & $\rho$ & SL $(\%)$ & $n$ \\
\hline All KBOs & Ampl. vs. $Q$ & -0.22 & 96.96 & 100 \\
& Ampl. vs. $H$ & 0.35 & 99.94 & 100 \\
\hline Classical & Period vs. $B-V$ & 0.66 & 98.49 & 17 \\
& Ampl. vs. $Q$ & -0.36 & 97.95 & 42 \\
& Ampl. vs. $H$ & 0.49 & 99.80 & 42 \\
& Ampl. vs. $i$ & -0.37 & 98.16 & 42 \\
& Ampl. vs. $e$ & -0.34 & 96.74 & 42 \\
\hline Hot & Period vs. $B-V$ & 0.66 & 98.11 & 15 \\
\hline Cold & Period vs. $Q$ & -0.80 & 94.97 & 7 \\
\hline Centaurs & Period vs. $B-V$ & 0.49 & 91.40 & 14 \\
& Period vs. $e$ & 0.47 & 94.04 & 18 \\
\hline Plutinos & Period vs. $H$ & -0.48 & 94.14 & 17 \\
\hline SDO's & Period vs. $V-R$ & -0.65 & 91.55 & 8 \\
& Ampl. vs. $H$ & 0.57 & 92.86 & 14 \\
\hline
\end{tabular}

respectively. On the other hand, if we fit Maxwellian distributions to the rotational frequencies, the mean of the distributions change slightly. The two main populations are separated here, giving a mean rotational period of $7.35 \mathrm{~h}$ (3.19 cycles/day) for the entire sample (upper panel), $7.71 \mathrm{~h}$ ( 3.11 cycles/day) for the TNOs (center panel), and $8.95 \mathrm{~h}$ (2.68 cycles/day) for the smaller Centaurs (lower panel).

As we can see in Fig. 4, there appears to be some fast rotators. In the center panel, for the TNOs, there is Ceto, $1996 \mathrm{TP}_{66}$, and $2004 \mathrm{GV}_{9}$ with single peak rotational periods of 2.21, 1.96, and $2.93 \mathrm{~h}$, which are quite short and unrealistic. The reason for this is that all 3 of the afore mentioned objects have light-curve amplitudes smaller than $0.15 \mathrm{mag}$ and were considered to have single-peaked light curves, whereas in reality they have doublepeaked light curves. A similar case occurs for the Centaur Chiron and 2002 GZ $_{32}$ (lower panel), which have a single peak rotational period of 2.96 and $2.9 \mathrm{~h}$, respectively, for the same reason.

\section{Correlations}

To search for correlations between different physical and orbital parameters (rotational period, peak-to-peak amplitude, semimajor axis, perihelion distance, aphelion distance, eccentricity, inclination), we use non-parametric statistical tests.

The presence of several periods and/or amplitudes for most of the objects, encouraged us to develop a customized code that randomly chooses one combination. The program created the individual combinations of period and amplitude pairs and calculated the correlation between one of these combinations (of period and amplitude) and the selected variable (e.g., colors, orbital parameters). The final choice of correlation, presented in Table 2, is the median among those correlations.

The statistical methods do not assume any particular population probability distribution, nor any functional shape. In particular, we compute all the correlations using the Spearman rank correlation, $\rho$ (Spearman 1904). This method is distribution-free and less sensitive to outliers than others methods. We studied the strength of the correlations by computing the Spearman coefficient $\rho$ and the significance level (SL). The $\rho$ coefficient has values between -1 and 1 . A correlation may exists if $\rho>0$, while $\rho<0$ implies a possible anti-correlation. A zero value means that there is no correlation at all. The significance of $\rho$ is 
measured by SL, the probability that the null hypothesis (samples not correlated) is not true. We consider that correlations or anti-correlations are strong when $\rho$ (in absolute value) is greater than 0.6 , a possible weak correlation exists when $\rho$ values are between 0.3 and 0.6 , and no correlations are present for values less than 0.3. The following criteria were used: SL greater than $95 \%=$ reasonably strong evidence of correlation; SL greater than $97.5 \%=$ strong evidence of correlation; and SL greater than $99 \%$ = very strong evidence of correlation. The interpretation of $\rho$, SL, and the graphical representation of the magnitudes allows us to detect possible correlations among the different magnitudes. Apart from these criteria, we consider that a possible correlation could exist when $\rho$ is greater than or equal to 0.3 and SL is greater than $80 \%$, in particular for samples of only a few objects. We emphasize that this particular threshold $(\rho>=0.3$; SL $>80 \%)$ provides only a hint of a possible correlation. We note that in the majority of these cases, the sample is so small, that more observations are required in some of the cases listed in Table 2.

The Spearman statistical analysis of the results was made by separating the dataset into different dynamical subgroups, i.e., classical objects, which exhibit orbits with moderate eccentricities; resonant objects, trapped by Neptune in mean motion resonances (the Plutinos being the most representative population, in the 3:2 resonance), and scattered disk objects (with orbits of high eccentricities and sometimes high inclinations, due to close encounters with the planet Neptune in the past). The classical group is sub-classified into two sub-groups: the so-called (dynamically) hot classical objects with orbital inclinations $>4.5^{\circ}$, and the (dynamically) cold classical objects, with inclinations $<4.5^{\circ}$. Finally, the Centaurs represent TNOs scattered towards the inner Solar System and they reside between the orbits of Jupiter and Neptune.

The analyzed parameters were the rotational period, lightcurve amplitude, colors $(B-V, R-I$ and $V-R)$, absolute magnitude $H$, and orbital parameters $(a, e, i, Q$ and $q)$. It should be mentioned that a data set of colors was presented in Santos-Sanz et al. (2009). All parameters were tested against all the other parameters in the entire sample and then in sub-populations. The corresponding correlation coefficients and significance levels are listed in Table 2, where only the relevant cases are shown. We made the test correlations with all the parameters in all the populations and then listed only the correlations that were found to be significant.

For the case of amplitude versus $H$, values of $\rho$ and SL for all the sample and in particular for the classical TNOs, clearly indicate that the smaller (and collisionally evolved) objects are probably more elongated than the larger ones. This could explain the larger amplitudes observed for smaller objects. In the classical population (39 objects), the value of $\rho=0.47$ and SL $=99.5 \%$ indicates a stronger correlation than the entire population with $\rho=0.40$ and $\mathrm{SL}=99.8 \%$. For the Centaurs and Plutinos, we do not find any evident correlation between these two magnitudes.

The particular case of $P$ versus $B-V$ correlations may suggest that objects with shorter rotation periods may have suffered more collisions (and thus have bluer surfaces) than objects with longer ones. The collisions could resurface the objects with fresh ices making them bluer and modifying the spins toward faster rotation periods. There is a strong evidence of a correlation in the classical subpopulation, although this sub-sample has fewer than 20 objects. More color determinations of objects with known periods would clearly be helpful, not only to confirm or exclude this possible correlation but to confirm whether there is some color variation in the surface of the body. The results obtained for

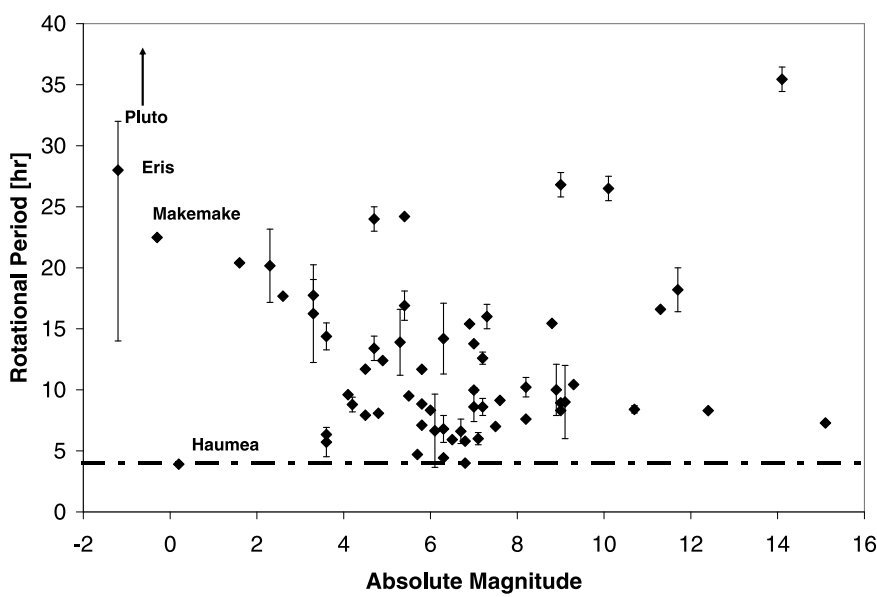

Fig. 5. Rotational Period against absolute magnitude for all the objects. The horizontal line at $4 \mathrm{~h}$ is the spin barrier mentioned in the text.

the $P$ versus $H$ relations of Centaurs (correlation) and Plutinos (anti-correlation) might be related to the different mean sizes of the Centaur and Plutino populations and could be related to a different primordial origin and/or evolution. In particular, physical parameters such as the color $B-V$ and rotational period are known only for brighter and larger objects. We, again note the low number of objects. We found some weak anti-correlations between the amplitudes and orbital parameters $Q$ and $e$, whose possible causes are not clear to us.

Finally, another interesting plot is the rotational period versus absolute magnitude (an indicator of the size), where we can see (Fig. 5) that a rotation barrier could exist close to $4 \mathrm{~h}$. All the points have an error bar that indicates the period determination error when the result is unique and a larger error when the period has several values in the literature. A typical example of multiple determinations in the literature is Eris, for which the period has been determined to be either $13.7 \mathrm{~h}$ (Duffard et al. 2008) or $25.92 \mathrm{~h}$ (Roe et al. 2008).

\section{Densities and internal structures}

With the rotational period sample, it is possible to derive some constraints on the density of the objects. Several authors have presented different estimations on TNO densities. Pravec \& Harris (2000) derived a simple expression that approximates the critical (minimum) period for a rotating body as a function of density and light-curve amplitude. This relation assumes a fluid body. Sheppard et al. (2008) used the periods and shapes (derived from light curves) to identify an apparent trend of larger (brighter) objects being denser. Finally, Tancredi \& Favre (2008) analyzed TNO densities and internal structures to determine whether TNOs could be dwarf planets.

We note that objects with radii $r>200 \mathrm{~km}$ have presumably never been disrupted by impacts (Davis \& Farinella 1997), but have probably been in some way fractured. If we assume that TNOs have relaxed to equilibrium shapes, then their rotation states can be used to place limits on their densities. The centripetal acceleration produced by self-gravity (bulk density) must be sufficient to hold the material together against the inertial acceleration due to rotation. Although TNOs consist of solid material, their presumably fragmentary structure validates the fluid approximation as a limiting case.

Densities have been proposed to be low, $600 \mathrm{~kg} / \mathrm{m}^{3}$ for $2001 \mathrm{QG}_{298}$ and in some cases, covering a wider range to higher 
Table 3. Some results using the simple model. Listed are the minimum and maximum rotational period that a Jacobi ellipsoid can rotate. The percentage of different kinds of objects (Jacobi/MacLaurin/non-equilibrium) are presented.

\begin{tabular}{lcrccccr}
\hline \hline $\begin{array}{l}\text { Density } \\
\mathrm{kg} / \mathrm{m}^{3}\end{array}$ & $\begin{array}{r}P[\min ] \\
\mathrm{h}\end{array}$ & $\begin{array}{r}P[\max ] \\
\mathrm{h}\end{array}$ & $\begin{array}{c}\Delta P \\
\mathrm{~h}\end{array}$ & $\begin{array}{c}P[\mathrm{eq}] \\
\mathrm{h}\end{array}$ & \% Jacobi & \% MacLaurin & \% No Eq. \\
\hline 400 & 9.99 & 10.91 & 0.92 & 8.88 & 6.51 & 22.04 & 71.43 \\
500 & 8.88 & 9.99 & 1.11 & 7.99 & 9.48 & 26.62 & 63.90 \\
600 & 8.27 & 8.88 & 0.61 & 7.27 & 7.53 & 36.34 & 56.12 \\
700 & 7.50 & 8.27 & 0.77 & 6.66 & 10.33 & 41.29 & 48.37 \\
800 & 7.05 & 7.74 & 0.69 & 6.31 & 10.38 & 46.26 & 43.35 \\
900 & 6.66 & 7.27 & 0.61 & 5.85 & 10.28 & 53.52 & 36.19 \\
1000 & 6.31 & 7.05 & 0.74 & 5.58 & 12.61 & 55.63 & 31.75 \\
1200 & 5.71 & 6.48 & 0.77 & 5.10 & 14.09 & 62.11 & 23.79 \\
1500 & 5.10 & 5.71 & 0.61 & 4.61 & 11.92 & 72.31 & 15.76 \\
2000 & 4.44 & 5.00 & 0.56 & 4.00 & 10.04 & 82.77 & 7.19 \\
2500 & 4.00 & 4.44 & 0.44 & 3.58 & 6.72 & 90.19 & 3.08 \\
3000 & 3.63 & 4.06 & 0.43 & 3.24 & 4.92 & 94.06 & 1.02 \\
\hline
\end{tabular}

densities of $2600 \mathrm{~kg} / \mathrm{m}^{3}$ as in $2003 \mathrm{EL}_{61}$. The lower density is similar to that inferred for some comets, which are objects two orders of magnitude smaller. Bulk densities substantially below that of pure water ice are usually taken to imply an ice-rich composition and porosity. It is expected that density variations are not only related to intrinsic compositional differences, but might be caused by variations in the porosity. On the other hand, for the dwarf planet class, porosity cannot be important. It needs to be mentioned that a density of $2600 \mathrm{~kg} / \mathrm{m}^{3}$ implies a rock/ice ratio of $\sim 85 / 15 \%$ when the internal structure is modelled (McKinnon et al. 2008).

\subsection{A simple Monte Carlo model}

Taking into account all the light curve amplitude and rotational period data presented, it can be seen that "flat curves" are dominant. As mentioned before, this could be because we are observing large presumably round objects. In the near future, more light curves should be obtained of smaller objects and the question may then arise of whether this tendency remains and how many non-flat curves are observed. To estimate how many nonflat curves are expected in an observed population, we developed a simple model.

The first step in our model is to assume a Maxwellian rotational frequency distribution such as that obtained in this work (Fig. 4), from the observational data. Then, each object from a set of 100000 of fixed density is randomly assigned to a rotational period from the distribution. The Maxwellian rotational frequency distribution adopted ranges from 8 to 0.5 cycles/day, with a bin of 0.1 and a peak at 3.11 cycles/day $(7.71 \mathrm{~h})$, in the observed distribution.

One important constraint of the model is that light curves are assumed to be related to the shape of the body. The model aims to determine the percentage of objects that create non-flat light curves due to elongated shapes. Other causes of brightness variation (not assumed here) are, for example, eclipses in binary objects or surface albedo variations.

For the model calculations, it is assumed that each object rotates in hydrostatic equilibrium and the Chandrasekhar formalism (Chandrasekhar 1987) is used. The questions that we try to answer is how many objects (of a given density) with different rotational periods could have an irregular shape with axes of lengths such that $a>b>c$ (a Jacobi ellipsoid), and how many objects acquire a MacLaurin shape (i.e., $a=b>c$ ). The final percentage of MacLaurin or Jacobi objects should be compared with the observed data.

Jacobi ellipsoids produce a non-flat light curve if the ratio of the axis lengths are greater than the photometric error. It is then necessary that the rotating axes are not aligned along the observer line of sight. MacLaurin objects will produce a flat light curve independently of the rotational axis direction. In all cases the objects are considered at opposition and in a simple rotation around the shortest axis. According to Burns \& Safronov (1973), rotational excitation damping times are orders of magnitude shorter than the age of the Solar System and are also orders of magnitude shorter than the typical time between collisions in the transneptunian region. In their study of ellipsoids, Molina et al. (2003) emphasized that damping times can be even shorter if one takes into account the energy dissipation caused by internal stresses. Thus, it is expected that the transneptunian objects must be purely in rotation around their principal axes of maximal moment of inertia, because the collisions that can excite their rotations take far longer to occur than the typical rotational excitation damping time. The TNOs have been found to be in pure rotation in other works, e.g., Ortiz et al. (2003a); Lacerda \& Luu (2003)

For all objects in each run, the dimensionless parameters $\Omega$ and $\Lambda$ (dimensionless angular velocity and dimensionless angular momentum) (Chandrasekhar 1987) were calculated. From these pairs of parameters and using Chandrasekhar's tables, it is possible to determine whether the object has a Jacobi or MacLaurin figure or if a non-equilibrium figure is possible. To obtain a complete sample of all possible densities, the model was run for several density values between 500 and $3000 \mathrm{~kg} / \mathrm{m}^{3}$.

\subsection{Results for the model}

For each density, the percentage of Jacobi, MacLaurin, and non-equilibrium figures were calculated and are presented in Table 3. In the case of the Jacobi ellipsoids, there is an interval of allowed rotational periods where the objects are in equilibrium. For example, for a density of $1000 \mathrm{~kg} / \mathrm{m}^{3}$, only $12.61 \%$ of the objects are Jacobi ellipsoids and they rotate with periods between 6.31 and $7.05 \mathrm{~h}$. For this same density, $55.63 \%$ of objects have a MacLaurin figure and the remaining $31.75 \%$ are non-equilibrium figures that were discarded from the sample.

In Fig. 6, the percentage of Jacobi, MacLaurin, and nonequilibrium objects is plotted against density for all the runs. As can be seen, when the density increases the percentage of 


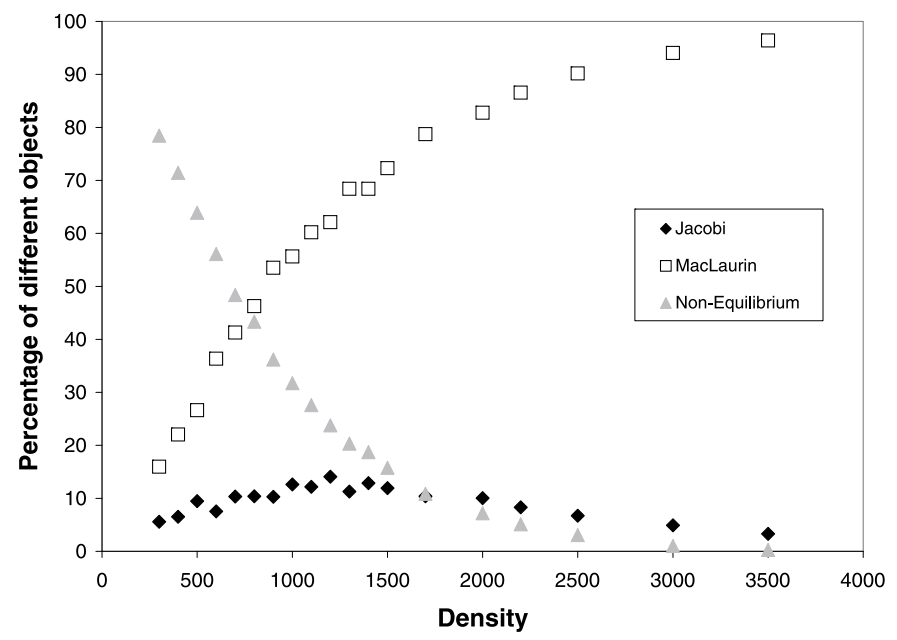

Fig. 6. Percentage of the 3 different kinds of object produced by the model against density (in $\mathrm{kg} / \mathrm{m}^{3}$ ). Jacobi ellipsoids are shown in black diamonds, MacLaurin spheroids in white squares and finally triangles are the non-equilibrium figures.

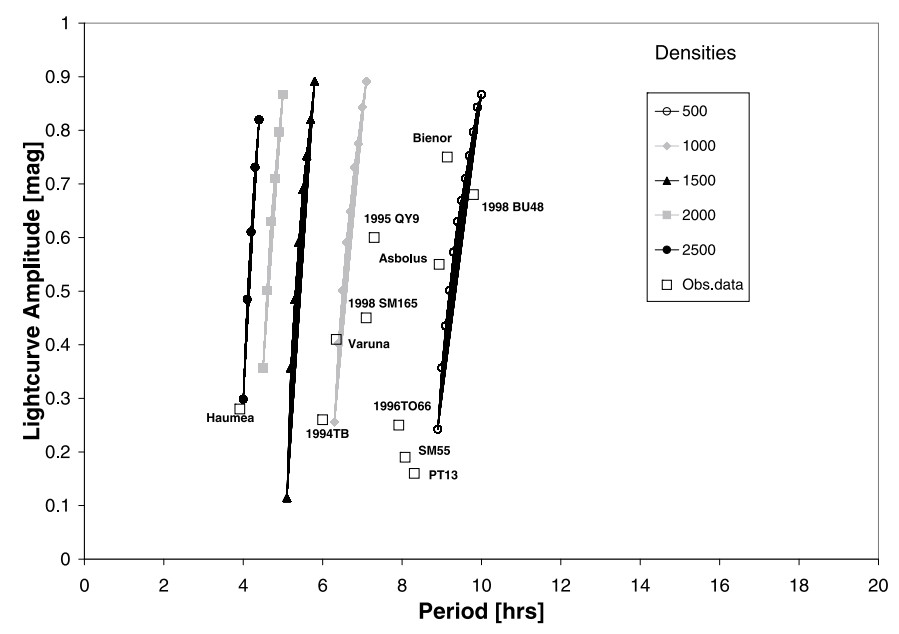

Fig. 7. light curve amplitude vs. rotational period for several model runs compared with some observational data. Densities are in $\mathrm{kg} / \mathrm{m}^{3}$.

MacLaurin spheroids and non-equilibrium figures, increase and decrease, respectively. The percentage of Jacobi ellipsoids has a maximum close to the densities of $1200-1300 \mathrm{~kg} / \mathrm{m}^{3}$.

The amplitude of the light curve of each synthetic object is then calculated from the axis ratio $b / a$ of the Jacobi ellipsoid. For a fixed density, the ratio $b / a$ of the ellipsoid increases with the rotational period. The obtained axis ratio was then modified by a random orientation of the rotational axis. This means that an elongated Jacobi ellipsoid would be seen pole-on and no lightcurve variation would be observable. In Fig. 7, the light curve amplitude for several runs of the model is plotted against rotational period. As can be seen, for each density there is an interval of allowed rotational periods for Jacobi ellipsoids. All the period-lightcurve amplitude pairs below that curve are allowed after the correction of the viewing angle. We note that the allowed rotational period interval for a lower density is longer than that allowed for higher densities, as expected. The rotational period interval becomes shorter when the density increases, in other words, the curve becomes steeper as the density increases.

Observed data is included in the figure to compare with the modelled data. From this figure, the density of a real object can be estimated by assuming that the objects are viewed equatorially. Haumea has a estimated density of $2500 \mathrm{~kg} / \mathrm{m}^{3}$, while most of the other selected objects have variations between densities 500 to $1000 \mathrm{~kg} / \mathrm{m}^{3}$.

Finally, in Fig. 8 the percentage of objects with different figures is presented for several densities. MacLaurin figures were added to the Jacobi ellipsoids in the case of small light curve amplitudes $(0.1 \mathrm{mag})$. Here it is assumed that if a light curve amplitude is detectable in a MacLaurin object, it will be smaller than $\sim 0.1 \mathrm{mag}$, because it will be produced by albedo features. For the different model runs (densities) we can observe that the percentage of objects with small light curve amplitude ( $0.1 \mathrm{mag}$ ) is always higher than the percentage of the more elongated ones, as expected. This percentage increases from $30 \%$ for a low density such as $500 \mathrm{~kg} / \mathrm{m}^{3}$ to more than $90 \%$ for a higher density like $2500 \mathrm{~kg} / \mathrm{m}^{3}$. On the other hand, as can be seen, the percentage of objects with large amplitudes is independent of density and is always smaller than $20 \%$. The percentage of MacLaurin objects sharply increases with density and this is the main reason for the differences between the curves at the left part of Fig. 8 (small light curve amplitudes).

In Fig. 8, the observational data are also plotted separated into two intervals of absolute magnitudes. The percentage of objects in the interval of $H$ between $[-1,5]$ and $[-1,7]$ is taken from Table 1. The behavior of both curves are the same as for the modelled ones. A large percentage of the objects have low amplitude light curves. Comparing this behavior with the modelled curves it is possible to estimate the mean density of the entire sample. As can be seen, the observational data agree with the output of the corresponding model run for $1000 \mathrm{~kg} / \mathrm{m}^{3}$ density. When smaller objects are included (black squares), the observed points are reproduced most accurately with an even lower density.

At the other side of the plot, it can be seen that observational data are lacking with respect to the model prediction. A blowup of the plot for the larger amplitudes is inserted in Fig. 8. As can be seen, some objects with larger light curve amplitudes are expected to be found.

To summarize, if hydrostatic equilibrium is considered with a Maxwellian rotational frequency distribution, a large percentage of "flat light curves" is obtained. This is because of the large percentage of MacLaurin figures added to the Jacobi ellipsoid with small amplitude (due to a small axis ratio or viewing angle). It is important to note that for the results mentioned above, only a distribution of rotational frequencies and a fixed density are assumed. No other physical variable is needed.

Finally, if a size distribution is included in the model, it is possible to compare the objects with the real sizes. For that, a cumulative surface density of TNOs was included as described in Petit et al. (2008) between $18<R<22$ mag. Assuming a mean albedo of 0.12 and mean distance of $40 \mathrm{AU}$, the smallest objects in this group have a radius of $86 \mathrm{~km}$. In other words, hydrostatic equilibrium seems to be valid for objects smaller than assumed before (Sheppard et al. 2008). This could be because the internal cohesion forces are smaller in TNOs than in asteroids. An estimated value of the tensile strength for this size that is overcome by the object's gravity, allowing the objects to behave like a fluid, is obtained from Eq. (3) of Thirouin et al. (2009). The tensile strength is only $0.1 \mathrm{MPa}$. This small tensile strength is much smaller than that of typical geophysical solids.

\section{Discussion}

With all the available data, we have compiled different histograms to see the distribution of physical parameters such as 


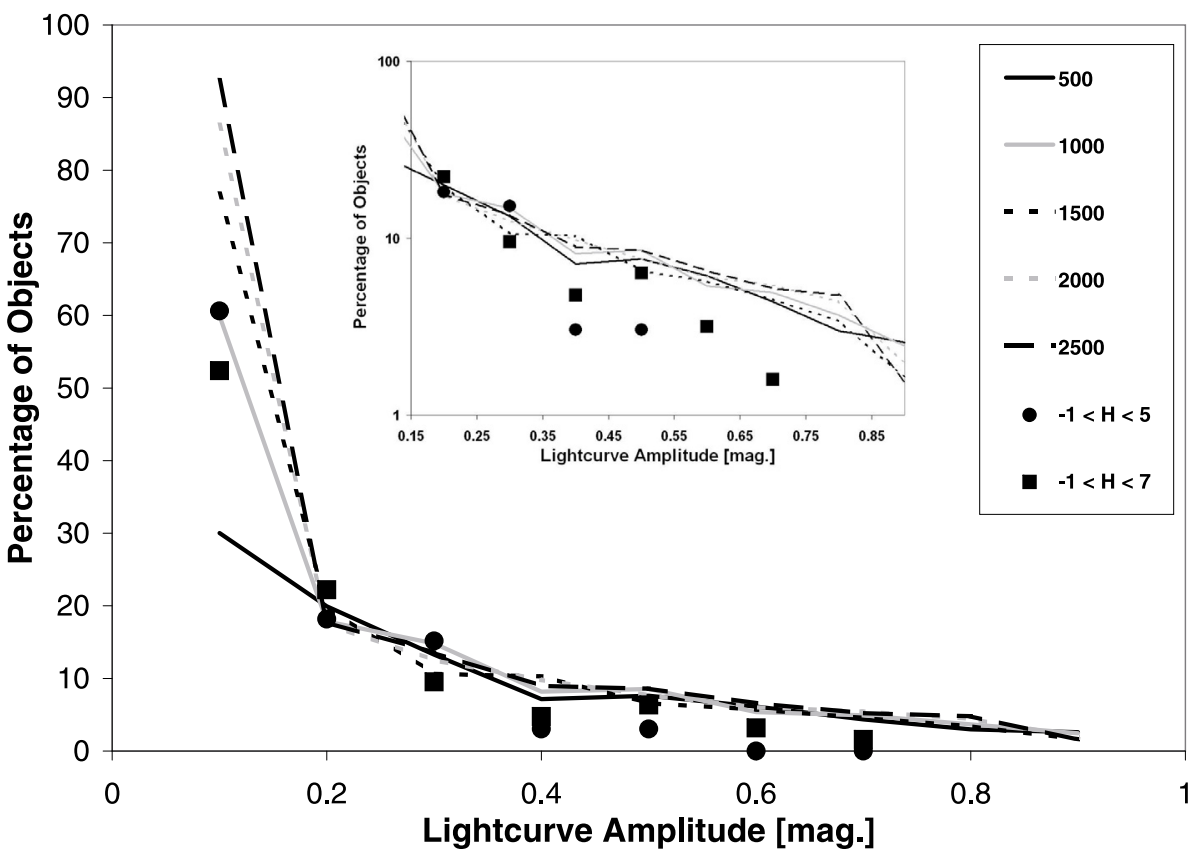

Fig. 8. Percentage of the objects having a certain light curve amplitude from the model for different densities. MacLaurin object and Jacobi ellipsoid were added together only for a light curve amplitude of 0.1 mag. Circles and squares are observational data using values of absolute magnitude between $[-1,5]$ and $[-1,7]$, respectively. rotational periods and light-curve amplitudes. With this larger number of light curves, the results obtained for the mean rotational period and peak-to-peak amplitudes are more reliable than in previous studies. The criteria to separate flat light curves from the others is important when selecting a single or double peak rotational period. As is demonstrated, the distribution of periods infers a lower limit (for a single-peak period) and upper limit (for double-peak periods). The result of the combination of both periods is a more reliable result. The threshold of 0.15 mag for the transition from the albedo-marking light curves to shape-induced light curves is somewhat arbitrary and all the results are based on this value. If a light curve amplitude magnitude of $0.10 \mathrm{mag}$ is considered the obtained results are similar, reaching a mean of $7.84 \mathrm{~h}$ and $7.12 \mathrm{~h}$ for all the sample and for only TNOs, respectively. The goodness of the Maxwellian fit is similar to the 0.15 mag case. However, if we use a threshold of $0.2 \mathrm{mag}$, the fit is degraded.

Why are their surfaces so uniform? The sample of flat light curves is dominated by larger objects. These bodies are expected to be round and in hydrostatic equilibrium. In these bodies, it is natural to ask: could the reason for flat light curves be the presence of a thin atmosphere or recent outgassing/resurfacing?

The environment in the trans-neptunian region might favor the formation of tenuous permanent or transient atmospheres. This idea is supported by several pieces of evidence such as (i) the intrinsic and/or induced activity on some bodies, such as in Chiron (Duffard et al. 2002) and other Centaurs. Activity has been reported for comets at large heliocentric distances and much beyond the water ice sublimation limit (Hainaut et al. 2000). (ii) Abundance of volatiles with sublimation temperatures comparable to the local temperature. (iii) The most important, the atmospheres of Pluto (Yelle \& Elliot 1997) and Triton (Yelle et al. 1995).

Our study is based on a photometric analysis, so we cannot determine the presence of atmospheres. The observational result is that most TNOs have small amplitudes. Taking the evidence together, it appears reasonable to expect the formation of thin gaseous and/or dusty atmospheres in large TNOs, because they would have the mass needed to avoid direct escape of materials into space. There is a study by Lykawka \& Mukai (2005) about the theoretical presence of an atmosphere according to several criteria and the possibility of the presence of a thin atmosphere in the studied bodies that takes into account volatiles such as methane, nitrogen, and carbon monoxide, the most common chemical species in the outer Solar System. It is noticeable that only TNOs with a diameter of $700 \mathrm{~km}$ (albedo criteria are explained in that article) can retain an atmosphere. The large self-gravity of the TNOs should allow them to remain close to hydrostatic equilibrium, retain extremely volatile ices, possibly have tenuous atmospheres, and be differentiated. Thus, the surfaces as well as the interior physical characteristics of the largest TNOs may differ significantly from those of the smaller objects.

A nearly uniform surface may be explained by an atmosphere that is frozen onto the surface of the objects when close to aphelion. The atmosphere, such as Pluto's, may become active when near perihelion, effectively resurfacing itself every few hundred years. Taking into account all the known light curves with known amplitudes and the work of Lykawka \& Mukai (2005), it is possible to identify possible candidates to be spectroscopically observed by searching for spectral signatures of an atmosphere.

The transient atmospheres and atmospheric freeze-out as a means of resurfacing and homogenization would apply to the largest objects only. An alternative scenario might be the collisional resurfacing, which may explain other phenomena in the transneptunian belt (Gil-Hutton 2002; Gil-Hutton et al. 2009).

In the model presented here, for different runs of only one density, the percentage of MacLaurin figures is always superior to $50 \%$ for densities higher than $900 \mathrm{~kg} / \mathrm{m}^{3}$. As can be seen in Fig. 8, the percentage of observed objects with light curve amplitudes smaller than $0.2 \mathrm{mag}$ is always $70 \%$. When compared to real data, it needs to be mentioned here that this sample is biased as observers tend to publish light curves with large amplitudes and easy-to-measure rotational periods.

According to our simple model, lower mean densities are obtained when we analyze the sample with the largest size span, from $H=[-1,7]$, compared to the interval $H=[-1,5]$. Hence, it appears that the "small" objects are also in hydrostatic equilibrium, but have lower densities than the largest objects. Therefore, an obvious refinement of our model could be 
to incorporate a size-dependent density together with the size distribution of the bodies whose brightness variability has been studied. Other improvements in the model could be the use of non-random spin axis orientations to test the effect of different spin axis distributions.

Despite the simplicity of our model, its results are compatible with observations. Currently, the only input of the model is a Maxwellian rotational frequency distribution, but the real distribution might differ from a Maxwellian, since this function is representative of a collisional equilibrium population. However, the true scenario might be represented by a combination of a collisional evolved populations and a more primordial one. Therefore, an additional improvement of the model would be the possibility to include different rotational frequency distributions.

As a caveat to the densities derived here, we can mention the work of Holsapple (2001) and Holsapple (2004) who determined the spin limits of bodies using a model for solid bodies without tensile or cohesive strength. That theory included the classical analysis of fluid bodies given by MacLaurin, Jacobi, and others as a special case. For the general solid bodies, it was shown that there exists a very wide range of permissible shapes and spin limits, and explicit algebraic results for those limits were given. Holsapple (2007) extended these analyse to include geologicallike materials that also have tensile and cohesive strength. In that paper, the author asked whether larger bodies such as the TNOs should have relaxed fluid shapes or might have shapes that are allowable by granular zero cohesion material? The important result is that if they are not governed by fluid theory, the mass and shapes that have been inferred could be substantially incorrect (Holsapple 2007).

Finally, we must mention that the search for correlations between light curve variables and orbital parameters is hampered by bias and selection effects (Binzel et al. 1989), and our conclusions in this respect are weak. However, the correlations between light curve parameters and physical properties such as colors and size that we derived are less affected by bias and we believe are reliable.

\section{Conclusions}

We have analyzed the light-curve data of Thirouin et al. (2009) and others in the literature to derive light-curve parameters, which have been compiled here. We have presented the most complete distribution of rotation periods and amplitudes so far. For the rotation periods, the mean value obtained by a Maxwellian fit in rotational frequencies is $7.71 \mathrm{~h}$ (3.11 cycles/day) considering only the TNOs, $8.95 \mathrm{~h}$ ( 2.68 cycles/day) considering only Centaurs, and 7.35 h ( 3.19 cycles/day) considering all the sample altogether. These results were obtained by taking into account the criteria of considering a single peak light curve for those objects with amplitudes lower than 0.15 mag and a double peak light curve for the more elongated ones.

This mean rotational period is slightly longer than the mean value for the largest Main Belt asteroids, $6.9 \mathrm{~h}$ (Binzel et al. 1989), but we state that this value may be affected by observational biases. It is important to stress that in photometry devoted to the study of the rotational properties of minor bodies, there exists and important observational bias towards shorter periods and higher amplitude light curves. How this bias can be estimated remains an open problem, but it certainly depends on the number of objects for which a period is obtained. Some steps in this direction were already taken by Thirouin et al. (2009).
Concerning other biases, if observational time for larger telescopes is granted then smaller TNOs will be accessible for the determination of the light curves, and the sample will not only increase in size but the bias in size will become less important.

One of the most important correlations found in this work is the light curve amplitude and absolute magnitude of all the sample of KBOs. This correlation is not a surprise and is probably related to the most collisionally evolved small population, which should be more elongated objects. On the other hand, the less collisionally evolved population (larger and then brighter objects) is the more numerous one in our sample ( $60 \%)$, so we caution the reader that some of our results could be somewhat biased. Another interesting correlation is the rotational period and $B-V$ color, which could indicate the age of the surface (younger surfaces being bluer). This correlation appears to be consistent with the idea that the more collisionally evolved objects spin faster and have bluer surfaces.

A simple shape model has been developed that is able to reproduce some results on the relative percentage of presence of MacLaurin/Jacobian figures, when assuming hydrostatic equilibrium. In the entire sample, less than $12 \%$ of the objects in hydrostatic equilibrium have a Jacobi shape. Most of the objects have MacLaurin figures that have small light curve amplitudes (because of albedo marks). The model that best fits the observations requires densities between 1000 to $1200 \mathrm{~kg} / \mathrm{m}^{3}$. We must mention that as was highlighted in Thirouin et al. (2009) there is an observational bias in the percentage of low amplitude light curves, which means that perhaps $75 \%$ of the objects have low light curve amplitudes. This implies that the mean density is likely somewhat higher, around $1500 \mathrm{~kg} / \mathrm{m}^{3}$ (see Fig. 8).

For plausible albedos of 0.04 to 0.20 , the absolute magnitude range $H=[-1,7]$ includes objects with diameters as small as $\sim 120 \mathrm{~km}$. Those objects would also be in hydrostatic equilibrium. We end with the provocative thought that the great majority of observed TNOs would qualify to be dwarf planets, becuase they appear to meet the hydrostatic equilibrium condition.

Acknowledgements. RD acknowledges financial support from the MEC (contract Juan de la Cierva). This work was supported by contracts AYA2008-06202C03-01, AYA2005-07808-C03-01 and P07-FQM-02998. We thank the referee for his/her constructive and helpful comments.

\section{References}

Bauer, J. M., Meech, K. J., Fernández, Y. R., Farnham, T. L., \& Roush, T. L. 2002, PASP, 114, 1309

Bauer, J. M., Meech, K. J., Fernández, Y. R., et al. 2003, Icarus, 166, 195 Belskaya, I. N., Ortiz, J. L., Rousselot, P., et al. 2006, Icarus, 184, 277 Benavidez, P. G., \& Campo Bagatin, A. 2009, Planet. Space Sci., 57, 201 Binzel, R. P., Farinella, P., Zappala, V., \& Cellino, A. 1989, in Asteroids II, ed. R. P. Binzel, T. Gehrels, \& M. S. Matthews, 416

Buie, M. W., \& Bus, S. J. 1992, Icarus, 100, 288

Buie, M. W., Tholen, D. J., \& Wasserman, L. H. 1997, Icarus, 125, 233 Burns, J. A., \& Safronov, V. S. 1973, MNRAS, 165, 403

Bus, S. J., Bowell, E., Harris, A. W., \& Hewitt, A. V. 1989, Icarus, 77, 223 Chandrasekhar, S. 1987, Ellipsoidal figures of equilibrium (New York: Dover) Choi, Y. J., Brosch, N., \& Prialnik, D. 2003, Icarus, 165, 101

Chorney, N., \& Kavelaars, J. J. 2004, Icarus, 167, 220

Collander-Brown, S. J., Fitzsimmons, A., Fletcher, E., Irwin, M. J., \& Williams, I. P. 1999, MNRAS, 308, 588

Collander-Brown, S. J., Fitzsimmons, A., Fletcher, E., Irwin, M. J., \& Williams, I. P. 2001, MNRAS, 325, 972

Consolmagno, G. J., Tegler, S. C., Rettig, T., \& Romanishin, W. 2000, in BAAS, 32,1032

Davies, J. K., McBride, N., Ellison, S. L., Green, S. F., \& Ballantyne, D. R. 1998a, Icarus, 134, 213

Davies, J. K., McBride, N., Green, S. F., et al. 1998b, Icarus, 132, 418

Davies, J. K., Tholen, D. J., Whiteley, R. J., et al. 2001, Icarus, 150, 69

Davis, D. R., \& Farinella, P. 1997, Icarus, 125, 50 
Degewij, J., Tedesco, E. F., \& Zellner, B. 1979, Icarus, 40, 364

Dotto, E., Perna, D., Barucci, M. A., et al. 2008, A\&A, 490, 829

Duffard, R., Lazzaro, D., Pinto, S., et al. 2002, Icarus, 160, 44

Duffard, R., Ortiz, J. L., Santos Sanz, P., et al. 2008, A\&A, 479, 877

Farnham, T. L. 2001, Icarus, 152, 238

Farnham, T. L., \& Davies, J. K. 2003, Icarus, 164, 418

Fernandez, J. A., \& Ip, W.-H. 1984, Icarus, 58, 109

Gaudi, B. S., Stanek, K. Z., Hartman, J. D., Holman, M. J., \& McLeod, B. A. 2005, ApJ, 629, L49

Gil-Hutton, R. 2002, Planet. Space Sci., 50, 57

Gil-Hutton, R., Licandro, J., Pinilla-Alonso, N., \& Brunetto, R. 2009, A\&A, 500, 909

Gomes, R., Levison, H. F., Tsiganis, K., \& Morbidelli, A. 2005, Nature, 435, 466

Gutierrez, P. J., Ortiz, J. L., Alexandrino, E., Roos-Serote, M., \& Doressoundiram, A. 2001, A\&A, 371, L1

Hainaut, O. R., Delahodde, C. E., Boehnhardt, H., et al. 2000, A\&A, 356, 1076

Hoffmann, M., et al. 1992, in Observations and Physical Properties of Smal Solar System Bodies, ed. A. Brahic, J.-C. Gerard, \& J. Surdej, Proc. Liege International Astrophys. Colloq., 30, 203

Holsapple, K. A. 2001, Icarus, 154, 432

Holsapple, K. A. 2004, Icarus, 172, 272

Holsapple, K. A. 2007, Icarus, 187, 500

Jewitt, D. C. 2004, From cradle to grave: the rise and demise of the comets (Comets II), 659

Jewitt, D., \& Luu, J. 1993, Nature, 362, 730

Kern, S. D. 2006, Ph.D. Thesis, Massachusetts Institute of Technology

Kern, S. D., \& Elliot, J. L. 2006, Icarus, 183, 179

Kern, S. D., McCarthy, D. W., Buie, M. W., et al. 2000, ApJ, 542, L155

Lacerda, P., \& Luu, J. 2003, Icarus, 161, 174

Lacerda, P., \& Luu, J. 2006, AJ, 131, 2314

Lacerda, P., Jewitt, D., \& Peixinho, N. 2008, AJ, 135, 1749

Lin, H.-W., Wu, Y.-L., \& Ip, W.-H. 2007, Adv. Space Res., 40, 238

Luu, J. X., \& Jewitt, D. C. 1998, ApJ, 494, L117

Lykawka, P. S., \& Mukai, T. 2005, Planet. Space Sci., 53, 1319

Magnusson, P. 1991, A\&A, 243, 512

McKinnon, W. B., Prialnik, D., Stern, S. A., \& Coradini, A. 2008, Structure and Evolution of Kuiper Belt Objects and Dwarf Planets (The Solar System Beyond Neptune), 213

Molina, A., Moreno, F., \& Martínez-López, F. 2003, A\&A, 398, 809

Morbidelli, A., Levison, H. F., Tsiganis, K., \& Gomes, R. 2005, Nature, 435, 462

Moullet, A., Lellouch, E., Doressoundiram, A., et al. 2008, A\&A, 483, L17

Mueller, B. E. A., Hergenrother, C. W., Samarasinha, N. H., Campins, H., \& McCarthy, D. W. 2004, Icarus, 171, 506

Ortiz, J. L., Gutiérrez, P. J., Casanova, V., \& Sota, A. 2003a, A\&A, 407, 1149

Ortiz, J. L., Gutiérrez, P. J., Sota, A., Casanova, V., \& Teixeira, V. R. 2003b, A\&A, 409, L13

Ortiz, J. L., Sota, A., Moreno, R., et al. 2004, A\&A, 420, 383
Ortiz, J. L., Gutiérrez, P. J., Santos-Sanz, P., Casanova, V., \& Sota, A. 2006, A\&A, 447, 1131

Ortiz, J. L., Santos Sanz, P., Gutiérrez, P. J., Duffard, R., \& Aceituno, F. J. 2007, A\&A, 468, L13

Osip, D. J., Kern, S. D., \& Elliot, J. L. 2003, Earth Moon and Planets, 92, 409

Peixinho, N., Doressoundiram, A., \& Romon-Martin, J. 2002, New Astron., 7, 359

Petit, J.-M., Kavelaars, J. J., Gladman, B., \& Loredo, T. 2008, Size Distribution of Multikilometer Transneptunian Objects, ed. M. A. Barucci, H. Boehnhardt, D. P. Cruikshank, \& A. Morbidelli, 71

Pravec, P., \& Harris, A. W. 2000, Icarus, 148, 12

Rabinowitz, D. L., Barkume, K., Brown, M. E., et al. 2006, ApJ, 639, 1238

Rabinowitz, D. L., Schaefer, B. E., \& Tourtellotte, S. W. 2007, AJ, 133, 26

Rabinowitz, D. L., Schaefer, B. E., Schaefer, M., \& Tourtellotte, S. W. 2008, AJ, 136, 1502

Roe, H. G., Pike, R. E., \& Brown, M. E. 2008, Icarus, 198, 459

Romanishin, W. \& Tegler, S. C. 1999, Nature, 398, 129

Romanishin, W., Tegler, S. C., Rettig, T. W., Consolmagno, G., \& Botthof, B. 2001, Proceedings of the National Academy of Science, 98, 11863

Rousselot, P., Petit, J.-M., Poulet, F., Lacerda, P., \& Ortiz, J. 2003, A\&A, 407, 1139

Rousselot, P., Levasseur-Regourd, A. C., Muinonen, K., \& Petit, J.-M. 2005a, Earth Moon and Planets, 97, 353

Rousselot, P., Petit, J.-M., Poulet, F., \& Sergeev, A. 2005b, Icarus, 176, 478

Santos-Sanz, P., Ortiz, J. L., Barrera, L., \& Boehnhardt, H. 2009, A\&A, 494, 693

Schaefer, B. E., \& Rabinowitz, D. L. 2002, Icarus, 160, 52

Sheppard, S. S. 2007, AJ, 134, 787

Sheppard, S. S., \& Jewitt, D. 2004, in BAAS, 36, 1081

Sheppard, S. S., \& Jewitt, D. C. 2002, AJ, 124, 1757

Sheppard, S. S., \& Jewitt, D. C. 2003, Earth Moon and Planets, 92, 207

Sheppard, S. S., Jewitt, D. C., Trujillo, C. A., Brown, M. J. I., \& Ashley, M. C. B. 2000, AJ, 120, 2687

Sheppard, S. S., Lacerda, P., \& Ortiz, J. L. 2008, Photometric Lightcurves of Transneptunian Objects and Centaurs: Rotations, Shapes, and Densities (The Solar System Beyond Neptune), 129

Spearman, C. 1904, Am. J. Psych., 100, 441

Tancredi, G., \& Favre, S. 2008, Icarus, 195, 851

Tegler, S. C., Romanishin, W., Stone, A., et al. 1997, AJ, 114, 1230

Tegler, S. C., Romanishin, W., Consolmagno, G. J., et al. 2005, Icarus, 175, 390

Thirouin, A., Ortiz, J. L., Duffard, R., et al. 2009, submitted

Tholen, D. J., \& Buie, M. W. 1990, in BAAS, 22, 1129

Trilling, D. E., \& Bernstein, G. M. 2006, AJ, 131, 1149

Tsiganis, K., Gomes, R., Morbidelli, A., \& Levison, H. F. 2005, Nature, 435, 459

Yelle, R. V., \& Elliot, J. L. 1997, Atmospheric Structure and Composition: Pluto and Charon, ed. S. A. Stern, \& D. J. Tholen, 347

Yelle, R. V., Lunine, J. I., Pollack, J. B., \& Brown, R. H. 1995, in Neptune and Triton, ed. D. P. Cruikshank, M. S. Matthews, \& A. M. Schumann, 1031 